\title{
Dramodwyr rhwng dau fyd: Aled Jones Williams a Sergi Belbel ${ }^{1}$
}

Nid yw edrych tua Chatalwnia yn beth newydd i academyddion yng Nghymru. Yn wir, mae’n rhywbeth a wneir yn fynych mewn cyd-destunau sosioeithyddol a gwleidyddol. 'Nôl yn 1998 amlygodd R. M. Jones potensial y gymhariaeth hon mewn pennod yn ei gyfrol Ysbryd y Cwlwm:

[M]i ddywedwn mai Catalunya [o blith y cenhedloedd lleiafrifol y mae'n cyfeirio atynt yn y gyfrol] yw'r un a all roi'r arweiniad trefniadol yng Ngorllewin Ewrob ar hyn o bryd, a hynny oherwydd nerth economaidd y boblogaeth sy'n siarad yr iaith ynghyd â phenderfynolrwydd yr adfywiad. Mae gan genhedloedd o'r fath wybodaeth arbennig am fywyd, a dylai'r hyn sy ganddyn nhw i'w ddweud fod o ddiddordeb cyffredinol, ac nid lleol yn unig [. . . . Os bydd ganddyn nhw, ar y llaw arall, y mentrusrwydd i feddwl ac i deimlo am fêr eu profiad eu hun, yna fe all fod ganddyn nhw rywbeth i'w ddweud o arwyddocâd treiddgar i bawb. Eu gwahaniaeth fydd eu grym. ${ }^{2}$

Fodd bynnag, digon prin yw’r gymhariaeth hon ym maes y celfyddydau, a phrinnach fyth yn nhermau'r theatr Gymraeg gyfoes. ${ }^{3}$ Nod yr ysgrif hon yw amlygu'r potensial i ehangu gorwelion cymharol ym maes beirniadaeth y ddrama Gymraeg a hynny trwy graffu ar waith dau ddramodydd cyfoes, dau a ystyrir yn ddramodwyr blaenaf eu cenhedloedd a’u cenhedlaeth, sef Aled Jones Williams o Gymru a Sergi Belbel o Gatalwnia. ${ }^{4}$ Gyda hynny, cyfeirir at waith ymchwil perthnasol ym maes y theatr gyfoes yng Ngalisia, yr Alban ac Iwerddon.

Beth felly yw'r tebygrwydd rhwng y ddau ddramodydd? Dechreuaf trwy ddyfynnu disgrifiad Sharon G. Feldman o waith Belbel, disgrifiad a fyddai'n taro deuddeg i ddarllenwyr sy’n gyfarwydd â gwaith Williams; 'As for Belbel, the role that verbal communication may play in apprehending reality has always been a matter of investigation and even a point of contention. ${ }^{5}$ Yn wir, amlyga’r dyfyniad un llinyn cyswllt ymhlith nifer rhwng gwaith y ddau, sef eu hoffter, a dry ar adegau'n obsesiwn, o gwestiynu rôl iaith fel cyfrwng i adlewyrchu ein bywydau. Ys dywed Williams:

Pam ydw i’n mwydro fy mhen efo geiriau pob munud? I beth? Rhywbeth ansad yw iaith. Does dim dichon ymddiried mewn geiriau. Mae rhywbeth yn disgyn drwy ogr geiriau o hyd: y 'peth' pwysicaf ran amlaf. Siomiant ydynt. ${ }^{6}$

Canlyniad y pethau 'sy’n disgyn drwy ogr iaith o hyd' yw diffyg cyfathrebu sydd, yn achos Belbel a Williams, yn troi'n boen, boed hynny’n boen o ganlyniad i fin cyllyll geiriau neu'n boen corfforol 
sy’n deillio o fethiant iaith. Yn wir, dadleua Feldman mai'r term 'Theatr Poen’ sydd fwyaf addas wrth ddisgrifio theatr Belbel: '[i]n essence, his theatre is about pain, for it is within the realm of anguish and affliction that the fissure between the visible and the invisible, sign and affliction, becomes most unmistakably apparent'. ${ }^{7}$ Yr hyn sy’n llechu rhwng y gweladwy a'r anweladwy a rydd rym i waith Belbel, disgrifiad sydd unwaith yn rhagor, yn taro deuddeg wrth feddwl am waith Williams, dadl a ddaw'n fwy eglur yng nghorff yr ysgrif hon. ${ }^{8}$

Disgrifir y boen yng ngwaith Belbel gan Feldman fel 'a pain that is symptomatic of our contemporary culture, a pain that invokes the presence of death and reminds us of the finitude of our existence’. 9 I Feldman, y syniad o ‘cultural aphasia’ yw’r allwedd i ddeall gwaith Belbel a’r boen a fynegir ganddo. Caiff y gair ‘affasia’ ei ddiffinio fel yr ‘[a]nallu i lefaru neu ddeall iaith o ganlyniad i glefyd neu niwed i’r ymennydd' gan Geiriadur Prifysgol Cymru. ${ }^{10}$ Â Ann Laura Stauler ati i ddisgrifio affasia fel a ganlyn:

a dismembering, a difficulty speaking, a difficulty generating a vocabulary that associates appropriate words and concepts with appropriate things. Aphasia in its many forms describes a difficulty retrieving both conceptual and lexical vocabularies and, most important, a difficulty comprehending what is spoken. ${ }^{11}$

Â Feldman hithau ymlaen i ddiffinio affasia diwylliannol yn nhermau, 'an inability to express verbally the conditions of a culture that has become morally bereft, ethically corrupt, spiritually dispossessed, and wholly void of compassion'. ${ }^{12}$ Gellid dadlau bod affasia diwylliannol yn ganlyniad anochel a chyffredin pan fydd un wlad rymus yn trefedigaethu un wannach, hyd yn oed pan fo’r wlad honno wedi ennill annibyniaeth, fel sy'n wir yn achos Iwerddon. Ymddengys nad yw'r ffenomen hon yn unigryw i Gatalwnia gan fod Cathy Lenny yn gofyn y cwestiwn isod mewn perthynas â'r theatr gyfoes yn Iwerddon:

If audiences live in a state of inbetween-ness: between history and virtuality, identity and performativity, narrative and intertextuality, spontaneous action and self-conscious reflexivity, hierarchized structure and postmodern playfulness, between the pre-modern and the postmodern, how does contemporary theatre explore their quandary? ${ }^{13}$ 
Er mai mewn perthynas â'r theatr yn Iwerddon y mae Lenny’n codi'r cwestiwn uchod, cwestiwn sydd hefyd yn cysylltu â’r affasia diwylliannol y mae Feldman yn ei ddisgrifio ydyw; hynny yw, mae Lenny’n gofyn sut y mae dramodwyr i ymateb i'r penbleth o fod 'rhwng, dau, air, rhwng, dau, olau, rhwng dau, lanw, rhwng, dau, fyd', chwedl y bardd o ddramodydd yn y bryddest a gipiodd y goron iddo yn Eisteddfod Genedlaethol 2002 ‘Awelon’. ${ }^{14}$ Y mae’r cyflwr hwn o ‘inbetween-ness’ yn lleisio profiad sy’n gyffredin i genhedloedd ôl-drefedigaethol. Yn wir, gellir gweld sawl cysylltiad â’r cyflwr hwn o fod rhwng gwahanol fydoedd a Thrydydd Gofod Homi Bhabha. ${ }^{15}$ Yn y gofod hwn, crëir a hyrwyddir hybridedd, term arall y mae Bhaba yn ei ddefnyddio mewn perthynas ag ôldrefedigaethedd, sef y gofod hwnnw rhwng y trefedigaethwyr a’r trefedigaethedig. ${ }^{16}$ Esbonia Bill Ashcroft, Gareth Griffiths a Helen Tiffin y syniad hwn ymhellach, 'hybridity commonly refers to the creation of new transcultural forms within the contact zone produced by colonization'. ${ }^{17}$ Term i ddisgrifio canlyniad yr ‘inbetween-ness’ ydyw hybridedd, felly, wrth i amrywiol fydoedd orgyffwrdd â’i gilydd. Trwy fynegi'r profiad hybrid hwn gwêl academyddion megis Peter Childs a R. J. Patrick Williams gyfleoedd newydd i symud grym o'r trefedigaethwyr i ddwylo'r sawl a drefedigaethwyd. ${ }^{18}$ Yn hyn o beth, byddai modd cymhwyso cwestiwn Lenny a holi sut y mae Williams a Belbel fel dramodwyr cyfoes yng Nghymru a Chatalwnia yn ymateb i'r 'rhyngdod' hwnnw. Credaf fod geiriau Williams isod yn llwyddo i daflu goleuni pellach ar y cyflwr hwn o fod rhwng sawl peth, yr hyn a fynegir yn sgil affasia diwylliannol, gan amlygu bod y term yn un addas i ddisgrifio’i waith ei hun, dramodydd y 'llais crin':

Gwn wedi’r Somme ac Auchwitz a Hiroshima nad oes dichon dal gafael mewn Duw allan yn fan'cw sy'n ymyrryd. Mae'r llun yma'n deilchion ynof a rhyngom. Clywn y darnau'n crensian o dan ein traed wrth gerdded drwy ein byd. . .Ond sydd eTo [sic] ynof, trwof. Fel gwrid. Fel cofio. Fel atgof. Fel anghofio. Fel cysgod yn lledu ar y pared. Fel drafft o dan ddrws. Ar y ffin wyf fi. Rhwng Duw nad oes dichon iddo fod wedi Auschwitz. A’r Adlais Arall ynof i gyd. Yn llais crin yng ngaeaf geiriau. ${ }^{19}$

Mae Williams a Belbel ill dau yn ysgrifennu o safbwynt dramodwyr sy’n byw mewn cenhedloedd sy’n amddifad o foesau, tosturi a chysylltiad ysbrydol. Mae’r ddelwedd o Dduw hollalluog bellach wedi ei chwalu ers erchyllterau'r ddau ryfel byd yn achos Williams a’r frwydr i warchod iaith a 
diwylliant dan yr unben Francisco Franco yn achos Belbel. Yn yr ysgrif hon, archwiliaf y modd y mae goblygiadau hynny’n atseinio yn nramâu'r ddau mewn cyfnod lle nad yw geiriau'n ffrwythlon bellach. Yn wir, â Williams mor bell â chyfeirio at y cyfnod hwn fel cyfnod y gaeaf geiriau, tra bod David George a John London wedi disgrifio Belbel fel 'a man that is fascinated by words but yet mistrusts them. ${ }^{20}$ Yr adwaith tuag at y byd di-foes hwn, byd lle y mae ffydd yn pylu a moesau'n frau yw’r affasia diwylliannol sy’n brigo i’r wyneb yng ngwaith Belbel a Williams. Yn yr ysgrif hon dadleuir mai dyna'r allwedd i ddeall sut a pham y mae nifer o linynnau yn cysylltu Williams a Belbel ynghyd.

\section{Dramodwyr blaenaf eu cenhedloedd}

Yn rhan nesaf yr ysgrif eir ati i esbonio pam fod Williams a Belbel yn cael eu hystyried yn ddramodwyr blaenaf eu cenhedloedd a'u cenhedlaeth.

Er mwyn cynnig syniad o apêl a dylanwad Belbel, aeth un adolygydd mor bell â dweud yn y papur newydd Catalaneg Avui, 'Sergi Belbel yw enw’r theatr Gatalaneg'. ${ }^{21}$ Dywedwyd hyn yn nyddiau cynnar llwyddiant Belbel. Roedd y llwyddiant hwn yn cynnwys ennill gwobr y Marques de Bradomin yn 1986 am ei ddrama Calidoscopis, gwobr ar gyfer dramodwyr dan ddeg ar hugain mlwydd oed. Aeth Belbel ymlaen i gipio rhestr hir o wobrau eraill i’w hychwanegu at y rhestr hon ac fe berfformiwyd ei ddramâu mewn nifer o wledydd gan gynnwys yr Almaen, De America a Ffrainc. $^{22}$ Ers hynny, perfformiwyd nifer o’i ddramâu gan Teatre Nacional de Catalunya, Forasters ac A la Toscana yn eu plith, a rhwng 2005 a 2013 bu'n gyfarwyddwr artistig ar y theatr honno. Mewn modd tebyg, cafodd Williams ei ddisgrifio gan yr academydd a'r adolygydd Roger Owen fel 'llais newydd mwyaf arwyddocaol y theatr Gymraeg yn y blynyddoedd diweddar' ${ }^{23}$ Fel Belbel, perfformiwyd rhai o ddramâu Williams, sef Iesu! a Pridd gan Theatr Genedlaethol Cymru. Awgrymodd Steve Blandford fod Iesu! ymhlith cynyrchiadau pwysicaf a mwyaf llwyddiannus Theatr Genedlaethol Cymru. ${ }^{24}$ Mae’r ffaith i Williams ddod i’r brig yng nghystadlaethau'r Fedal 
Ddrama ym 1994, 1995 a 1996 a hynny’n groes i gonfensiynau’r cyfnod hefyd yn tystio i’r awch a fu am ei ddramâu. Yn eu beirniadaeth ar y gystadleuaeth yn 1995 esboniodd Carys Edwards, Huw Williams a Graham Laker fod i ddrama Williams ‘Pêl Goch’ elfen o dwyll: ‘[a]r yr wyneb, mae hi’n ymddangos fel cawdel anhrefnus sydd wedi llifo o’r isymwybod yn ddireol [. . .]', ond, wrth ddarllen ymhellach esboniodd y beirniaid iddynt gael eu plesio gan themâu perthnasol ac iaith brydferth y ddrama. ${ }^{25}$ Cyfeirio y mae'r beirniaid yma at barodrwydd Williams i chwarae ag iaith, ffurf a themâu er mwyn ceisio mynegi'r profiad rhwystredig o fyw yng Nghymru heddiw. Canlyniad hynny’n aml yw mynegi'r syniad o fyw mewn byd hybrid y mae’r beirniaid yn ei ddisgrifio fel 'byd rhwng dau fyd' ${ }^{26}$ Roedd hi'n amlwg i'r beirniaid fod gan y dramodydd 'rywbeth i'w ddweud wrthym am ein byw a'n bod fel pobl y dwthwn hwn yn ein hanes', ffactor sydd wedi cyfrannu at ei apêl theatrig a syniadol i gynulleidfaoedd Cymru. ${ }^{27}$

Mewn modd tebyg, nododd Joan de Sagarra fod gan Belbel neges ar gyfer ei wlad, a’i fod, yn wir, yn symud y wlad honno, pobl y cyrion, tuag at ymdeimlad o normalrwydd. Nododd i Belbel ei helpu i gymodi â’r theatr yng Nghatalwnia. Dywed ei fod erbyn hyn yn teimlo:

a sense of reconciliation with this country, with this small patria that is the Catalan stage, and it not only makes me feel like a normal person, a normal reader, spectator in a normal country, which one would hope is definitely normalized, but also moves me. And the only thing that pains me is not being able to share my emotion with some very dear people from the small patria that is the Catalan stage, in which they always believed and who today are no longer with us. ${ }^{28}$

Diddorol yw sylwi ar y modd y mae Sagarra uchod yn cyfeirio at y theatr yng Nghatalwnia fel 'patria’, hynny yw, fel gwlad. Sylwer hefyd ar ddefnydd helaeth Sagarra o’r gair 'normal’ yn y dyfyniad sy’n cloriannu dylanwad Belbel. I Sagarra, mae’r sylw rhyngwladol a roddwyd i Belbel wedi normaleiddio'i genedl, ei iaith ac wedi dyrchafu ei theatr i sylw’r byd: 'Here to be “normal“ is to move from the periphery to the center [sic.], to be regarded as valid rather than illicit, and to be visible and vociferous - even obvious and everyday' ${ }^{29}$ Gellid dadlau i Belbel greu gofod hybrid sy’n 
mynegi’r profiad o ‘inbetween-ness’ y mae Cathy Lenny yn ei gyfeirio ato, y cyflwr o ymwrthod â chael eich pegynnu a chreu, yn hytrach, y Trydydd Gofod hwnnw. Yn hynny o beth, yr hyn a wna Belbel a Williams yn y bôn yw ymrafael â'r 'power to narrate or to block out narratives from forming', a thrwy hynny mae'r rhai a drefedigaethwyd yn rhydd 'to assert their own identity and the existence of their own history'. ${ }^{30}$ Canlyniad yr hybridedd hwn ydyw'r ymdeimlad o banig o du'r trefedigaethwyr, 'the margin of hybridity, where cultural differences "contingently" and conflictually touch, becomes the moment of panic which reveals the borderline experience'. ${ }^{31}$ Hynny yw, yn hytrach na chael eu gosod ar y naill begwn neu’r llall (pegwn y trefedigaethwyr neu'r trefedigaethedig) crëir cyfuniad gwahanol, anodd i’w ddiffinio, sy’n esbonio, o bosibl pam fod cynifer wedi ei chael hi’n anodd i osod gwaith Belbel a Williams mewn carfan benodol. O ran hynny, ‘[e]u gwahaniaeth yw eu grym’, chwedl R. M. Jones.. ${ }^{32}$ Nid yw’r profiadau a fynegir gan Williams o fod 'rhwng' dau fyd, o fod ar y ffin, yn rhai unigryw mewn gwirionedd. Esbonia Bhabha fod y Trydydd Gofod yn amlygu safbwyntiau ymylol a thrwy hynny crëir gofodau lle y gellir 'emerge as others of ourselves'. ${ }^{33}$ Potensial y theatr mewn cenhedloedd fel Cymru a Chatalwnia yw helpu'r fath bobl i dyfu tuag at normalrwydd cenedlaethol, 'instead of being obstructed, silenced or relegated to the margins of exile, the recess of memory, or the darkness of invisibility'. ${ }^{34}$ Oherwydd mae i'r theatr rym y tu hwnt i'r llwyfan ac adeilad y theatr yn unig, fel y noda David Adams yn Stage Welsh: Nation, Nationalism and Theatre: The Search for Cultural Identity: 'the Great National Theatre Debate is really about more than theatre. It is about the state of Wales, about cultural identity, about nationalism and internationalism. But debaters only talk about theatre'. ${ }^{35}$ Wrth gymharu gwaith y ddau ddramodydd dan sylw â'i gilydd, gobeithir amlygu cyn bwysiced yw edrych y tu hwnt i’n cymdogion yn Lloegr a chymharu ein sefyllfa â chenhedloedd diwladwriaeth eraill sy’n ceisio dod o hyd i'w hunaniaeth.

Dramodwyr rhwng deufyd: rhwng yr abswird a dychwelyd at iaith 
Roedd llwyddiant eisteddfodol Williams ym maes y ddrama yn groes i dueddiadau'r cyfnod. Wrth graffu'n fanwl ar gystadlaethau drama'r Eisteddfod Genedlaethol rhwng 1960 a 2015, sylwyd bod dramâu abswrdaidd wedi mwynhau cryn dipyn o lwyddiant rhwng 1960 ac $1965 .{ }^{36}$ At hynny, roedd gweithiau dramodwyr o weddill Ewrop, Pinter, Beckett a Ionesco yn eu plith, yn ffasiynol. Trwy archwilio sylwadau’r beirniaid eisteddfodol yn ystod y cyfnod dan sylw (1960-2015), daeth yn amlwg fod cyw-ddramodwyr Cymru yn awyddus i efelychu camp a sioc gychwynnol dramâu W. S. Jones a Gwenlyn Parry. Serch hynny, cythruddwyd beirniaid gan gynigion gwan a oedd yn tybio mai rhywbeth hawdd i’w efelychu oedd y ddrama abswrdaidd. Nid Theatr yr Absẃrd ei hun oedd wrth wraidd rhwystredigaeth beirniaid y gystadleuaeth, ond yn hytrach ddiffyg crefft a diffyg ystyriaeth i’r cymhelliad cymdeithasol hanfodol sydd ei angen i lunio dramâu o’r fath. Hanfod dramâu abswrdaidd y chwedegau oedd yr argyfwng gwacter ystyr hwnnw a olygai nad oedd y ddrama destunol glasurol yn medru cyfleu baich profiadau'r dramodwyr a'u rhwystredigaeth bellach, a heb yr ymdeimlad cychwynnol hwnnw, ynghyd â dealltwriaeth dda iawn o egwyddorion a rheolau drama, nid oedd modd i’r efelychwyr ddenu canmoliaeth y beirniaid o gyflwyno iddynt ddelweddau symbolaidd a chymeriadau di-blot. Fe’u beirniadwyd yn llym am ddiffyg crefft. Ystyrier sylw John Gwilym Jones yn ei feirniadaeth ar gystadleuaeth y Ddrama Hir yn Eisteddfod Genedlaethol Frenhinol Cymru Maldwyn a’r Cyffiniau, 1981:

Mae rhyw ffiloreg beryglus ar droed y dyddiau hyn sy’n cyhuddo gair mewn drama o fod yn ormes. Nid dweud a wneir y gellir creu sgwrs heb eiriau ond nad ydyw fawr o wahaniaeth sut eiriau ydynt, nad oes gofyn ymboeni i roi pleser mynegiant fel y cyfryw, mai'r hyn a ddywedir yn hytrach na'r ffordd y’i dywedir sy'n bwysig. Ond tybed nad yw synnwyr cyffredin heb sôn am barch i gelfyddyd yn ffieiddio'r fath safbwynt? Ffieiddio beth bynnag a wna pob dramodydd o bwys. Onid oes rhin arbennig heb sôn am swyddogaeth i ailadrodd, '’Ryn ni’n aros Godot' yn nrama Beckett? Geiriau cyffredin, ie, - a does dim o’i le yn hynny - ond geiriau wedi eu dewis yn ofalus i bwrpas arbennig. Ac nid yw o unrhyw wahaniaeth p'run ai trasiedi ai comedi ai ffars yw'r ddrama, heb hyn mae'n fethiant. ${ }^{37}$

Ymddengys fod rhai dramodwyr yng nghystadlaethau drama’r Eisteddfod Genedlaethol wedi camgymryd y ddrama abswrdaidd fel ffurf hawdd i'w hefelychu, heb roi ryw lawer o sylw i'r grefft 
gychwynnol gan gamgymryd chwarae geiriol dramodwyr abswrdaidd i fod yn ddim ond llifeiriant o eiriau disynnwyr. Anwybyddwyd hanfodion drama, rhywbeth y mae Bethan Jones a Siân Summers yn rhoi pwyslais arno yn eu beirniadaeth ar gystadleuaeth y Ddrama Hir Agored yn Eisteddod Genedlaethol Tyddewi, Sir Benfro, yn 2002:

Mae croesffrwythloni ac arbrofi - er mwyn ymestyn gallu'r ffurf i gyfathrebu'n ddilyffethair, yn oesol berthnasol ac yn gyffrous - yn beth iach, wrth gwrs. Ond mae'n rhaid wrth ymwybyddiaeth o'r ffurf gysefin fel sail i'r ymdrech o fentro. ${ }^{38}$

Williams oedd 'ond’ mawr y patrwm hwn y sylwais arno rhwng 1960 a 2015 - gan i’w ddramâu anghonfensiynol ddod i’r brig yng nghystadlaethau drama’r Eisteddfod Genedlaethol dair blynedd yn olynol. Y rheswm dros hynny yw am iddo, fel Belbel, gefnu, i raddau, ar y symbolaidd a dychwelyd at y testun. Wrth drafod gwaith Belbel, dywedodd Lluis Antonio Villena:

Ar adegau, mae'r theatr wedi ceisio ymatal rhag siarad, a gwneud pantomeim ac ystumiau yn lle. Rwyf wedi credu erioed mai dramâu sy'n rhoi pwyslais ar y testun yw'r dyfodol. Mae Sergi Belbel yn cytuno â fi. Mae Carícies yn gampwaith am ei bod yn siarad, yn awgrymu ac yn fud. ${ }^{39}$

Mae'n amlwg fod rhai beirniaid yng Nghatalwnia, yn ôl y dyfyniad uchod, fel sydd hefyd yn wir am Gymru, yn teimlo mai rhywbeth dros dro oedd y symbolaidd a’r abswrdaidd. Yn wir, dyna oedd bwriad y math hwn o theatr, fel yr esboniodd Martin Esslin, y gŵr a fathodd y term: ‘[y]esterday’s revolutionary techniques have become today’s conventions. ${ }^{40}$ Lleisiwyd y rhwystredigaeth hon yn fynych gan feirniaid y Fedal Ddrama, ynghyd â rhai o adolygwyr y dramâu abswrdaidd a lwyfannwyd gan y Theatr Genedlaethol, ynghyd â sylwadau nifer o feirniaid y theatr yng Nghatalwnia megis Enrique Centero a Carles Batlle i Jordà. ${ }^{41}$ Apêl dros dro ydoedd un Theatr yr Absẃrd, fel y rhagwelodd Gwyn Thomas ’nôl yn 1976, ‘[f]e all hyn [Theatr yr Absẃrd] fod yn chwyldro ym myd celfyddyd; eithr y mae’n amheus gennyf ai hynny ydi o'. ${ }^{42}$ Disgrifiodd Thomas ddramâu abswrdaidd megis '[c]amre mewn anghelfyddyd', gan ddadlau ymhellach fod y math hwn o theatr yn ddim ond anarchiaeth sy’n 'groes i'r broses o greu ac yn groes i’r egwyddor o drefn, ac y mae ceisio gwneud celfyddyd heb y nodweddion hyn mor amhosib â chael dŵr heb gyfuniad o ddwy 
ran o heidrojen i un o ocsijen. ${ }^{43}$ Yn 2008, dros ddeg ar hugain o flynyddoedd ers i Gwyn Thomas ddarogan fyrhoedledd apêl yr absẃrd, nododd Gareth Miles:

Ni fu dylanwad Theatr y Di-sens (cyfieithiad Saunders Lewis o Le Théâtre de l'Absurde) yn llesol i’r ddrama Gymraeg. Tybia dynwaredwyr Beckett, Pinter a Ionesco, yn enwedig rhai ifainc, nad oes raid i ddrama fod am ddim byd ac y gellir llunio un yn reit hawdd o gymeriadau haniaethol, sefyllfaoedd ffantasïol, deialog gwta, swta, ddisynnwyr a llond llwyfan o wacter ystyr. ${ }^{44}$

Er bod rhai beirniaid yn pwysleisio ei bod hi'n amser symud ymlaen, mae'n amlwg yn sgil sylwadau rhai beirniaid eisteddfodol megis sylwadau Mari Emlyn a Cefin Roberts yn 2010 bod nifer o ddramodwyr Cymru’n gyndyn i wneud hyn:

Roedd sawl un wedi dewis ysgrifennu yn arddull yr absẃrd ac felly, o bosib, dan yr argraff fod modd ymaflyd yn syth i lunio deialog heb feddwl am y darlun cyflawn a heb bori dros themâu a'u llinynnau storïol yn gyntaf. ${ }^{45}$

Nid beio Theatr yr Absẃrd a wna’r beirniaid. Yn hytrach, credant mai diffyg dealltwriaeth dramodwyr o’r grefft sy’n ofynnol i lunio drama sydd wrth wraidd y broblem.

\section{Apêl yr Absẃrd: Cymru a Chatalwnia}

Beth felly yw Theatr yr Absẃrd a beth oedd apêl natur symbolaidd yr absẃrd i’r ddwy genedl? Pam eu bod nhw mor gyndyn i symud ymlaen? Yn achos Catalwnia, bu’n rhaid troi at ddramâu symbolaidd yn ystod cyfnod yr unben Francisco Franco gan nad oedd hawl llwyfannu dramâu Catalaneg. Cynigiodd elfen symbolaidd y theatr abswrdaidd y cyfrwng perffaith i greu theatr yn ystod y cyfnod heriol hwnnw, yn wleidyddol ac yn artistig. ${ }^{46}$ Yn achos Cymru, roedd dramodwyr yn teimlo y dylid efelychu rhai o ddramodwyr mawr Ewrop tua diwedd y 1950au a dechrau'r 1960au, dramodwyr amrywiol a digyswllt ond rhai a gyflwynwyd ynghyd yn llyfr arloesol a dylanwadol Martin Esslin, Theatre of the Absurd (1961).

Nod dramodwyr Theatr yr Absẃrd oedd ymateb i erchyllterau'r ddau ryfel byd gan nad oedd y theatr ‘naturiolaidd’ yn medru cludo baich yr hyn yr oedd ganddynt i’w ddweud bellach. Ystyriai J.L. Styan ddramâu abswrdaidd yn ‘adwaith nihilaidd i’r erchyllterau diweddar, y siambrau nwy a 
bomiau niwclear rhyfel’. ${ }^{47}$ Ar ôl clec fawr y sioc gychwynnol, pylu a wnaeth apêl y ddrama abswrdaidd wedi hynny wrth i gynulleidfaoedd ymgyfarwyddo â’i hergyd. ${ }^{48}$ Roedd dramodwyr Cymru, fodd bynnag, yn gyndyn i symud ymlaen er gwaethaf sylwadau rhai beirniaid eisteddfodol, adolygwyr a beirniaid llenyddol megis Gwyn Thomas a Gareth Miles. Sylwodd Carles Batlle i Jorda ar duedd debyg yng Nghatalwnia. Ar ôl cyfnod Franco cafwyd cyfnod anghynhyrchiol ac roedd dramodwyr fel pe baent ar goll heb symbyliad creadigol er mwyn medru ymateb i greisis. ${ }^{49}$ Pam felly? Ystyrier adolygiad theatr Robert Morley, 'I have been brooding in my bath for the last hour and have come to the conclusion that the success of Waiting for Godot means the end of the theatre as we know it'. ${ }^{50}$ Efallai nad yw hi'n syndod fod dramodwyr mewn cenhedloedd llai megis Cymru a Chatalwnia yn awyddus i efelychu math o theatr a greodd gymaint o gyffro mewn cenhedloedd mawr. Yn wir, y mae’r awydd i efelychu, yn ôl meddylwyr megis Bhabha, yn greiddiol i’r berthynas drefedigaethol: 'mimicry emerges as one of the most elusive and effective stratergies of colonial power and knowledge'. ${ }^{51}$ Esbonia ymhellach, 'mimicry represents an ironic compromise[. . .]colonial mimicry is the desire for a reformed, recognizable Other, as a subject of difference that is "almost the same but not quite”, ${ }^{52}$ Caiff yr awydd hwn i ddynwared sylw gan R. M. Jones hefyd wrth iddo esbonio ei fod yn duedd gyffredin mewn cenhedloedd lleiafrifol; 'gall ymddangos i rywrai llai hyderus na’i gilydd mewn lleiafrif di-statws y dylid dynwared a chydymffurfio â'r norm hwnnw, norm sydd yn y bôn yn perthyn i sefyllfa bloc-pŵer’. Ond rhybuddia Jones yn erbyn hyn, gan fod 'holl blethwaith bywyd yn y lleiafrif bygythiedig yn wahanol'. ${ }^{53}$ Y mae Williams ei hun, yn ei nofel Ychydig Is Na'r Angylion yn tynnu sylw at awydd adolygwyr yng Nghymru i gysylltu gwaith dramodwyr â gwaith dramodwyr blaenllaw sy’n ysgrifennu’n Saesneg:

Fel y mae ambell un yn deud am ddramodydd o Gymro, y Beckett Cymraeg. Gan feddwl bod hyn yn rhywfath o ganmoliaeth. Ond sarhad ydy o. Fel petai'n rhaid ini wrth fagal o enw enwog o wlad arall i’n gneud ni'n ddilys. ${ }^{54}$

Mae Williams uchod yn adleisio'r syniad y mae Bhabha yn ei grybwyll, y syniad ein bod ni am fod yn debyg i’r sawl sy’n ein trefedigaethu, ac eto i gyd yr ydym yn crefu i fod yn wahanol. 
Gwelir yr un duedd yn Ngalisia, yn ysgrif Burghad Baltrusch. Cred Baltrusch fod yr ymserchu hyn yn yr absẃrd yn fodd o fynegi:

the existence of a pronounced political desire for progress and innovation in the Galician cultural polysystem. This Galicianizing impulse, predominantly nationalist in character, sought to overcome the impediments of diglossia, the inferiority complex created by Francoism, and a negative collective memory based on a history of poverty and emigration. $^{55}$

Gellid dadlau fod yr awydd hwn i arloesi, i feddwl am ffordd arall o fynegi eu hunain, hefyd yn un o ganlyniadau trefedigaethu ac yn fodd o geisio goresgyn affasia diwylliannol.

Cyfrwng defnyddiol yw Theatr yr Absẃrd felly i rai o ddramodwyr Galisia a Chymru a Chatalwnia fynegi eu rhwystredigaeth wyneb yn wyneb â’r hyn a alwodd Baltrusch yn gymhlethdodau israddoldeb ar sail eu hanes. ${ }^{56}$

Yn wahanol i Gwenlyn Parry a nifer o ddramodwyr amlwg Theatr yr Absẃrd, ni chafodd Williams ei effeithio’n uniongyrchol gan y rhyfeloedd byd. Mae David George a John London hefyd yn awgrymu bod y ffaith nad oedd Belbel wedi ysgrifennu yn ystod cyfnod Franco wedi ei alluogi i fod yn fwy mentrus o lawer o ran ffurf a chynnwys ei ddramâu. Dyna, yn eu barn nhw, sydd wedi cyfrannu at awydd y dramodydd i arbrofi a gwthio'r ffiniau. ${ }^{57}$ Dywed David George ymhellach yn ei gyfrol ar waith Belbel fod yr awydd i ddychwelyd at y testun, yn groes i ddramodwyr cyfnod Franco, yn arwydd ei fod yn fwy sicr a chyfforddus wyneb yn wyneb â’i genedl. ${ }^{58}$

Yn wir, y mae Belbel ei hun yn dweud, ‘os ydw i’n hoffi La Fura dels Baus [cwmni theatr stryd sy’n awyddus i wthio’r ffiniau ac sy’n rhoi pwyslais ar y ddelwedd yn hytrach na’r gair] a Molière fel ei gilydd, nid wyf yn fy ngwrthddweud fy hun. ${ }^{59}$ Esbonia George ymhellach:

It is as though Belbel were continually playing with form. In contrast to many dramatists from preceding generations, locked into a system of censorship, Belbel has no qualms about admitting his lucid approach to theatre: 'I think I want to play and why, shouldn't I if I'm given the chance? ${ }^{60}$ 
Gwelir yr awydd amlwg hwn i chwarae ac arbrofi trwy dynnu ar amrediad o fudiadau a ffurfiau theatrig yng ngwaith Williams hefyd. ${ }^{61}$ Mae Chwilys ganddo yn enghraifft dda o ddrama sy’n gwthio ffiniau'r ffurf theatrig. Ceir dau ddiweddglo posib yn Chwilys a chaiff y diweddglo ei bennu gan yr actorion ar y noson. Mae hyn yn dwyn i gof ddrama gan Belbel Morir, sef drama mewn dwy ran, lle y ceir 'morir' (marw) lle mae’r cymeriadau’n marw, a ‘no morir' (peidio â marw), lle gwelir yr un golygfeydd eto ond gyda chanlyniad tra gwahanol wrth i'r cymeriadau lwyddo i ddianc rhag marwolaeth. Mewn cyfweliad gyda Nic Ros esboniodd Williams fod gweld Wrth Aros Godot, un o ddramâu amlycaf Theatr yr Absẃrd, wedi bod yn dipyn o drobwynt iddo ond yn yr un cyfweliad â'r dramodydd ymlaen i sôn am ddylanwadau eraill arno, gan ymhelaethu'n benodol ar ei hoffter mawr o King Lear, drama sy’n dilyn patrwm mwy clasurol o weld cymeriad yn ei ddigwydd, a gweld ‘dyn wedi dod lawr i’w elfennau, yn yr elfennau'. ${ }^{62}$

Er bod i ddramâu Williams a Belbel elfennau abswrdaidd, megis yr awydd i gwestiynu effeithlonrwydd iaith, cymeriadau sy’n fwy o deipiau na chymeriadau crwn a gyflwynir yn eu dramâu. Credaf fod eu gwaith yn cynrychioli shifft o fewn eu traddodiadau theatrig. Mae’r dyfyniad isod gan Feldman yn disgrifio’r shifft yng Nghatalwnia:

The theatre of the word returned with renewed energy, force, and prestige; however, once it "returned," it was not the same. Whereas the points of reference for previous dramatists had been Antonio Buero Vallejo, Bertolt Brecht, Arthur Miller, Eugene O’Neill, or Josep Maria de Sagarra, now they are Samuel Beckett, Thomas Bernhard, Edward Bond, Bernard-Marie Koltès, Tony Kushner, David Mamet, Heiner Müller, Harold Pinter, Sam Shepard, and Botho Strauss.

Rai blynyddoedd yn ôl, wrth iddi drafod gwaith Gwenlyn Parry, dadleuodd Elan Closs Stephens mai 'Gwenlyn fu'r bont rhwng y theatr Gymraeg a theatr yr absẃrd' ${ }^{63}$ Yn hynny o beth, gellid dadlau bod Williams a Belbel yn adeiladu ar Theatr yr Absẃrd trwy gyfuno elfennau clasurol a dylanwadau eraill, megis In-Yer-Face-Theatre, sy’n esbonio rhai golygfeydd amrwd sy’n digwydd yn nramâu Williams a Belbel. ${ }^{64}$ Gellid dadlau bod Williams a Belbel fel ei gilydd rhwng dau fyd o safbwynt arddull eu dramâu, rhwng dylanwad yr absẃrd a’r clasurol, y delweddol a’r geiriol a’u bod yn rhydd 
i dynnu ar amrediad o fudiadau hen a newydd er mwyn mynegi'r profiad o fyw yn eu cenhedloedd heddiw, cenhedloedd sydd rhwng dau fyd neu, yn hytrach, gyfresi o fydoedd cyferbyniol, rhwng darostyngiad a datganoli, a rhwng datganoli ac annibyniaeth.

\section{Iaith: 'Rhethreg a rhegfeydd a'r twll uffernol rhwng y ddau'}

Dyma enghraifft o ddau fyd arall y mae’n hawdd canfod tensiynau rhyngddynt yng ngwaith y ddau ddramodydd, tensiynau rhwng realiti a'r anallu i gyfleu’r realiti hwnnw trwy gyfrwng iaith. Yn wir, dyma un o hanfodion syniad Feldman o affasia diwylliannol. Mae dau o gymeriadau Williams yn ei ddrama ‘Pêl Goch’ yn cyfleu’r syniad i’r dim:

GWRAIG: Sgin ti 'im byd i' ddeud?

GWR: Methu 'i ddeud o ydw i.

GWRAIG: Iesu Grist mi ydan ni 'di mynd fel na fedrwn ni neud 'im byd fel cenedl ond pendilio rhwng rhethreg a rhegfeydd ... Rhethreg a rhegfeydd a'r twll uffernol rhwng y ddau. . . ${ }^{65}$

Archwilio methiant iaith y mae’r ddau ddramodydd, ac archwilio'r 'twll uffernol' rhwng yr hyn y maent am ei ddweud a’r hyn a ddwedir a wneir yma.

Cydnebydd Williams natur lithrig iaith a'r modd y mae’n amhosibl i eiriau gyfleu'r union brofiad neu deimlad dan sylw. Dengys y pytiau isod y modd y mae Belbel fel Williams yn mynegi'r rhyfeddod a'r amheuaeth ochr yn ochr â’i gilydd. Mae cymeriadau En Companyia d'abisme yn dadansoddi a chwestiynu ystyr geiriau'n fanwl:

MAN: 'To meet suddenly', 'surprised to meet', 'not knowing anything previously', 'chance meeting', nothing set out'...but what does it all mean?

YOUNGER MAN: And what does 'ambiguous' mean? And 'bored'. And 'most strange' MAN: Forgive me, but 'most strange' is only superlative of 'strange'[. . . ]

A chawn esiampl debyg yn 'Wal' gan Williams:

ALJI: Lapis Laswli . . .Maen iaspis a sardine. . .Yn debyg yr olwg arno i smaragdus... EDDY: Be ma’ hynny’n 'i feddwl? 
ALJI: Petha tlws

Jyst geiria

Bygro ystyr

Jyst enjoia’r geiria fel tasan nhw'n dda-da yn dy geg di. . . ${ }^{67}$

Gwelir sut mae’r ddau yn cwestiynu ystyr geiriau yn yr esiamplau uchod. Geiriau Alji sydd fwyaf cynrychioliadol efallai - 'bygro’r ystyr' - gan mai mwynhau sŵn a rhythm y geiriau a wna'r ddau. Yma, gwelwn brydferthwch iaith ac anallu'r iaith honno, er ei phrydferthwch, i ddweud rhyw lawer ‘fel tasan nhw'n dda-da' yn eu cegau. Mae iaith, fel y mae Feldman yn ei esbonio yn, ‘essentially ineffectual in apprehending reality’ ${ }^{68}$ Dyma enghraifft arall o waith Belbel sy’n cyfleu aneffeithlonrwydd iaith fel cyfrwng i gyfleu realiti. Dyma bwt o sgwrs y dyn a’r ddynes yn Tàlem:

The Woman: What then?

The Man: What?

The Woman: That's right, what?

The Man: What what?

The Woman: When will it be here?

The Man: When will it be here, then.

Pause. ${ }^{69}$

Sylwer nad yw’r un cwestiwn yn cael ei ateb uchod. Yn hytrach, ymatebir iddo drwy gyfrwng cwestiwn arall. Yn ei ragymadrodd i Dins la Seva Memòria, cymharodd J. Sanchis Sinisterra ddeialog rhythmig Belbel â cherddoriaeth geiriau Samuel Beckett yn ei ymgais i greu 'mater soniarus - rhythmig a ffonetig - iaith'. ${ }^{70}$ Gellid dweud yr un peth am yr enghraifft isod o 'Wal' gan Williams, drama lle mae'r berthynas rhwng ei chymeriadau yn annelwig:

EDDY: Chdi 'ta fi?

MUDANDOD.

EDDY: Chdi 'ta fi?

MUDANDOD.

EDDY: Chdi 'ta fi?

MUDANDOD.

ALJI: Meddylia am ffor arall o ddeud hynny nei di plîs? Plîs...

EDDY: Fi 'ta chdi?

ALJI: ’Na welliant!. . .Smwythach ar y glust rwsud. . . ${ }^{71}$ 
Eto ceir yr argraff mai ymhyfrydu yn sŵn a rhythm iaith y mae'r cymeriadau yma, am eu bod o bosibl, yn cydnabod mor gwbl aneffeithiol yw iaith i gyfleu syniad neu brofiad. Mae'r dyfyniad o Lysh isod yn awgrymu hynny ymhellach:

Ond y geiria - O! y geiria - yn rhydd o’r print yn gandryll yn y nghrebwyll i. Omphalos. Oubliette. Scybalum. Oxymoron. Y cwbwl ohony’ nhw wedi colli eu hystyron. Ac yn sefyll yn fan'no yn noethni eu sŵn. Fel bocsar wedi tynnu ei fenyg. ${ }^{72}$

Y mae'r syniad o sefyll yn noeth yng nghanol sŵn geiriau yn ddelwedd a welir yn fynych yng ngwaith Williams yn benodol. Ystyrier y dyfyniad isod o Oerfel Gaeaf Duw fel esiampl arall o hyn: ‘[s]iarad bymtheg yn dwsin w’t ti. Baricedio dy hun hefo geiria. T’yd allan o dy gamofflaj geiria a wynebu petha. ${ }^{73}$ Gwelir Williams yma yn ymwrthod â'r syniad o 'mimicry camouflage', chwedl Jacques Lacan, ‘[i]t’s not a question of harmonizing with the backround, but against a mottled background, of becoming mottled - exactly like the technique of camouflage practiced in human warfare'. ${ }^{74}$ Er ei fod yn symud rhwng cuddio o dan gwrlid geiriau a’u noethni yn fynych yn ei ddramâu, syniad sy’n apelio’n fawr at Belbel hefyd, mae ganddo awydd mawr i wynebu pethau. Isod, gwelwn hynny ar waith yn Tàlem wrth i Belbel bwysleisio mor anodd ydyw canfod y noethni, neu’r ystyr yng nghanol y geiriau weithiau:

The Woman: Well, it's certainly not going to come by itself, is it?

The Man: Of course not.

The Woman: You did say right now? Right now, yes? Right now? Well, it's already been 'right now' for the past two hours, hasn't it?, and 'right now' is already now, if I'm not mistaken, and it still hasn't come, it hasn't come, it hasn't come; at least, as far as I can see I can't see anything here, here, anything at all, anything at all, anything at all, oh oh oh we're going to end up without anything, tonight, tonight we'll have to sleep on the street if we go on like this, two hours like two idiots since they took the other one away, and there's still no sign of new one, is there? Oh, yes, yes, yes, I'm going I'm going I'm going, perhaps we'll be lucky but I'm still going, perhaps if I leave, your 'right now right now' will become a reality and, then!, what a stroke of luck and it comes at that very instant; now if that happens, it's not a good sign!, it will mean that it doesn't like me! I'm going. I'll die of embarrassment when they see me walk in. Two weeks late! The way I hurried them to to to to put it together (oh, is that how you say it?, put it together?). And what, well, then, you, what do you think?, shall I go? is it clear to you or or or or not, come on, what do you think? ${ }^{75}$ 
Er helaethrwydd y geiriau uchod yn Lysh ac yn Tàlem, nid oes yr un ohonynt yn gallu cyfleu'r hyn y maent am ei ddweud. Eto i gyd, perthyn tinc delynegol i iaith Williams sy’n llwyddo i grynhoi teimlad anodd ei fynegi i’r dim ar adegau. Gwelir enghreifftiau nodedig o hyn yn Anweledig gan Williams:

Tyd at y môr, Mi fendi'n fanno'.

Pryd, Dad? Pryd 'na'i fendio?

'Tria ddeud wrthai, 'mach i, sut beth ydy o?, meddai Huw'n dynerach.

Mae o fel bod mewn pictiwrs, medda fi, a neb yno yn y tywyllwch ond chdi yn gwatjad hen ffilm sâl. Dy holl fywyd di ydy’r ffilm. A fedri di ddim codi o dy set. Mae o fela. 'Ond ma pob ffilm yn dŵad i ben,' meddai Huw yn llythrennol fel arfar.

Neu mae o fel cerddad traeth yn y gaea' a chditha'n pigo llanast yr ha': coes lolipop yn fanna, chwartar pêl las yn colli 'i lliw fan draw, un flip-flop, wynab frizbee mewn gwallt o wymon, gwddw potal gwrw'n picio o'r tywod. Broc petha'. Mae o fela.

A ma Huw’n nodio’i ben fel petai o wedi dallt i’r dim. 'Yli! Mi na i banad.’ Medda fo. ${ }^{76}$

Mae gallu Williams i fynegi syniad trwy ddelweddau geiriol ymhlith y pethau y mae Gruffudd Eifion Owen yn ei ganmol yn ei adolygiad o Anweledig, a gwelir hynny ar waith yn bendifaddau. ${ }^{77} \mathrm{Yn}$ Anweledig mae yna ymgais drwy eiriau drwy wahanol ddelweddau, i wynebu pethau, i esbonio ofnau dyfnion, ac yn yr achos hwn yr iselder sy’n llethru'r wraig.

Gwelir peth tebyg yn Dins la Seva Memoria gan Belbel, wrth i un o gymeriadau’r ddrama sôn am y profiad o’i adnabod ei hun, ““Ef”, am y tro cyntaf ef oedd “ef”. “Ef” gair rwyt ti’n dechrau ei ddefnyddio o hyn ymlaen. ${ }^{78}$ Yr hyn a wna’r darnau mwy barddonol hyn yn y bôn yw pwysleisio ‘the shifting nature of dialogue’ yn ôl Maria Delgado a David George. ${ }^{79}$ Dyfais arall ydyw i amlygu twyll iaith, cyfrwng arall i leisio’r cwestiynau y mae Williams yn eu gofyn: ‘Fedri di ymddiried mewn iaith? Sut mae iaith, sut mae gair yn gallu cyfleu’r teimlad sydd y tu mewn i ti? Eto, mae’n rhaid i ti enwi’r teimlad, ond ydi hynny’n dal y teimlad? ${ }^{80}$ Eto i gyd, mae’r cyfan yn ymddangos yn sgyrsiol-ddealladwy, yn wahanol i iaith fwy eithafol abswrdaidd dramodwyr megis Samuel Beckett, er enghraifft, ac eto mae hyn oll yn fodd arall o bwysleisio twyll iaith. ${ }^{81}$ 
Yn aml iawn, caiff y gagendor rhwng iaith a’r syniad neu’r profiad y tu ôl iddo a’r tu hwnt iddo ei drosglwyddo’n bellter rhwng y cymeriadau, fel sy’n digwydd yn achos cymeriadau 'Pêl Goch’ neu 'Fel Stafell' gan Williams. Ystyrier yr enghraifft isod o 'Fel Stafell’:

GŴR: Lle fuos di?. . .Dw i 'di dy golli di. . .

GWRAIG: Do...

GWR: E's oria. . .

GWRAIG: E’s blynyddoedd. . .

SAIB. ${ }^{82}$

Mae’r un peth hefyd yn wir am nifer o ddramâu Belbel. Dengys deialog Marc a Joana o A la Toscana yr un pellter sy’n cael ei leihau i raddau, a’i ymestyn hefyd drwy iaith:

Marc: Wyt ti’n fy ngharu?

Joana: Ydw.

Marc: Wir?

Joana: Beth yw'r ots os yw'n wir? Ti’n marw. Yr hyn sy’n bwysig yw 'mod i’n ei ddweud e. A bod ti'n ei gredu.

Marc: Ie. (Saib.) Rwy’n ei gredu. Dyna’r unig gysur, ynte fe? (Saib.) Dwed wrtha i. Joana: Rwy'n dy garu. ${ }^{83}$

Ynghyd â gweld y gagendor sydd wedi tyfu rhwng Marc a Joana uchod, sylwer hefyd ar allu iaith i guddio realiti. Roedd y weithred o ddweud ei bod hi'n ei charu yn ddigon i leddfu ofnau Marc, er nad yw Joana'n llefaru o ryw argyhoeddiad mawr. Tystio i boen cyfathrebu a wnawn yn nramâu'r ddau ddramodydd hyn.

Canlyniad arall y diffyg cyfathrebu yw bod iaith yn troi’n fwy na gagendor yn unig, ac yn troi’n drais. Yn achos Belbel mae David George nodi fel hyn; '[v]iolence is closely linked to the concept of the dysfunctional family or couple, particularly in Carícies, Morir, Forasters and Mòbil'. ${ }^{84}$ Ystyrier y cyfnewid isod rhwng dau gymeriad yn Morir, er enghraifft. Yn yr olygfa hon mae’r fam a’r ferch yn gwybod yn union sut i frifo ei gilydd a defnyddiant iaith 'fel cyllyll i frifo', chwedl John Gwilym Jones. $^{85}$

Merch: Dyw gwm cnoi ddim yn mygu'r teimlad llwglyd achos ddoe o’n i’n bwyta gwm cnoi drwy'r dydd ac mi oeddwn i bron â marw'n moyn bwyd.

Mam: A beth sy'n bod arnat ti? 
Merch: Dim. Am fy mod i am fynd ar ddeiet. Dywedodd ffrind wrtha‘ i fod gordewdra’n rhywbeth yr wyt ti'n ei etifeddu.

(Mae'r Fam a'r fin rhoi slap i'r Ferch) ${ }^{86}$

Hawdd yw gweld rhyfeddod Belbel parthed gallu dirgryniadau tannau'r llais i wneud niwed.

Gwelir hefyd sut y mae’r dweud a'r gwneud yn un, fel y mae Feldman yn ei esbonio

ymhellach; 'In terms of aesthetic construction, quite often, the action not only precedes the word; the action is the word. ${ }^{87}$ Mae sylw Feldman yn adleisio sylwadau Tudur Hallam mewn perthynas ag egwyddor 'Parler c'est agir', d’Aubignac. Meddai; ‘[y] dweud yw’r gwneud', hynny yw, yn y geiriau y mae'r digwydd, nid yn unig y symudiadau corfforol ar y llwyfan. ${ }^{88}$ Gwelir hyn ar ei orau yn Anweledig gan Williams, monolog heb symud corfforol. Yn wir, mae'r cymeriad yn gaeth i'w chadair, ac eto gellid dadlau ei bod hi’n un o ddramâu grymusaf y dramodydd ac ynddi’r ‘digwydd’ mwyaf. Yn Anweledig, y dweud yw’r gwneud.

Modd arall y mae’r dramodwyr dan sylw yn dangos gallu iaith i frifo ydyw trwy dwyll iaith.

Daw'r enghraifft isod o Lysh. Yma gwelwn Ifor yn celu ei salwch y tu ôl i’r tasgau a’r esgusodion, y we o gelwyddau y mae’n ei chreu, a hynny drwy gyfrwng barddoniaeth iaith bob dydd:

Dwi'n mynd i nôl torth, cariad. A dwi'n dychwelyd diwrnod wedyn. Lle ydw-i? Yn y ngwely! Ond tydw-i ddim yn sâl. Be dwi'n 'neud yn fan hyn? Pryd ddois i i fan hyn? Pryd oedd ddoe? Ac fel gwyrth mae'r dorth wedi troi'n botal fodca wag wrth'n ochor i. A dwi'n codi rwsud. Mynd allan rwsud. I nôl torth arall. Torth o wydr. ${ }^{89}$

Dyma enghraifft o’r celwydd sy’n llechu rhwng geiriau. Profir hyn wrth i weithredoedd Ifor fynd yn groes i’r hyn y mae’n ei ddweud. Mae hyn hefyd yn wir am gymeriadau Belbel. Mae problemau dyrys megis trais yn y cartref a materion rhywioldeb yn cael eu gwrthgyferbynnu â thasgau bob dydd mewn dramâu fel Carícies a Forasters. Ystyrier yr enghraifft isod o Carícies:

Young Woman: Excuse me just a minute.

Young Man: What?

She punches him in the stomach and knees him in the groin. He falls to the ground.

Young Woman: We've run out of oil.

Young Man: Oh. ${ }^{90}$ 
Mynegir math arall o fwlch yn ‘Ta-ra Teresa’ sef y bwlch rhwng y gwir a’r darlun ohono. Gwelwn agendor rhwng y darlun y mae’r prif gymeriad Robat Hefin yn ceisio ei gyfleu a’r hyn a ddigwyddodd mewn gwirionedd. Pwysleisir yr abswrdiaeth drwy'r hyn a ddywed Robat Hefin ei hun, wrth iddo ymguddio, ‘dodjian pethau’ mewn geiriau:

Llefydd i ddengid iddyn nhw oedd y tu mewn i iaith. Dodjian pethau tu mewn i'r geiria. Mae brawddeg dda yn medru mynd â chi i le arall, diarth. Oherwydd dwi'n rhydd ymysg y geiria. Mi oedd bod tu mewn i'r iaith Gymraeg fel bod mewn syrcas. Rhyfeddod un gair ar deitrôp brawddeg yn peri i chi ddal 'ch anadl mewn syfrdan. Acrobats geiria'n tymblo i ddelwedd na feddylioch chi 'i bod hi hyd yn oed yn bosibl gneud y ffasiwn beth. Dau air yn clownio hefo’i gilydd. Yr holl giamocs ar drapîs yr iaith. . .91

Ceir gagendor tebyg yng ngwaith Belbel fel yng ngherdd Williams 'Parlimpsest', 'twyllo' a 'maglu' y mae geiriau, a chodi’n fi/ gwahanol’: ‘O gaglau’r brawddegau agori le â dwylo iaith i fi arall ddod trwyddo?’.92 Fodd bynnag, nid yw’r cymeriadau mor sicr o’r gwirionedd a’r hunandwyll yn ei ddramâu ef, megis yn A la Toscana lle mae dryswch yn aml rhwng yr hyn sydd wedi digwydd mewn gwirionedd a breuddwyd neu ffantasi un o gymeriadau'r ddrama. ${ }^{93}$ Yn A la Toscana ymddengys fod nifer o farwolaethau'n digwydd yng nghwrs y ddrama, ond dim ond un farwolaeth sy'n digwydd mewn gwirionedd. Mae’r gweddill yn digwydd yn nychymyg Marc, y prif gymeriad. Gellid cymharu'r dryswch hwn â'r ddrama Merched Eira gan Williams, lle mae’r ddau gymeriad mewn byd rhwng dau fyd arall, yn y cyflwr hwnnw rhwng byw a marw. ${ }^{94}$ Mae marwolaeth a natur annelwig marwolaeth o ddiddordeb mawr i'r ddau ddramodydd. Yn wir, yn Merched Eira y mae un o’r cymeriadau'n dweud am farwolaeth: ‘Mae marwolaeth O! mor hyblyg'. Dyma syniad sy’n apelio at Belbel a Williams fel ei gilydd. ${ }^{95}$

Gwna Williams a Belbel ill dau ddefnydd helaeth o’r fonolog yn eu dramâu. Esboniodd Williams fod y ffurf yn apelio gan ei bod yn gyfrwng i archwilio gwrthdaro mewnol:

Gwrthdaro mewnol ydi o, a dweud y gwir yn onest. Pobl sy’n ymlafnio â nhw eu hunain. Rhwng - unwaith eto - y goleuni a'r tywyllwch yma, rhwng y da a'r drwg, rhwng byw a dim byw. Ymlafnio mewnol - yn fan'no mae'r gwrthdaro gen i. Mae rhyw fath o. . . 
rhywbeth rhwng ffrae fewnol ambell dro sydd, efallai, yn troi’n ddeialog dro arall. [. . .] Lleisiau mewnol yn tynnu fan hyn a fan draw. Y llais mewnol ydi'r un. ${ }^{96}$

Er bod ambell ddrama megis Despres de la Pluja a 'Wal' yn ymddangos fel dramâu ac iddynt ddeialog, mae modd ystyried cymeriadau'r dramâu hynny fel un cymeriad a holltwyd yn sawl rhan er mwyn eu harchwilio’n fanylach. Mae’r ffaith bod rhan gyntaf enwau Alji ac Eddy o’u cyfuno yn sillafu 'Aled' yn cynnig rywfaint o sail i'r ddadl honno. Mae pob cymeriad fel pe bai'n ffurfio rhan o syniad neu drafodaeth. Gwelir hynny yn Lysh hefyd, wrth i bob un o gymeriadau'r ddrama gyfrannu at ein darlun o’r hyn yw bod yn alcoholig. A dywed Feldman am Belbel: ‘[p]lot and characterization are frequently established through subjective fragments and shreds, not through objective/naturalistic/psychological approaches. ${ }^{97}$ Nid cael cymeriadau hollol grwn sy’n bwysig i'r dramodwyr hyn. Fel y mae Nic Ros yn ei esbonio: ‘Er cystal cyfle a gynigia cymhlethdod y cymeriadau i actorion, nid yw’r portread a gynigia Williams yn real nac yn grwn. ${ }^{\text {98 }}$ Er bod digon o gig ar eu hesgyrn i ennyn cydymdeimlad y gynulleidfa, cymeriadau sy’n amlygu profiad penodol a natur heriol cyfathrebu ar lafar ydynt. Yn hynny o beth, datgymalu cymeriadau y mae’r dramodwyr hyn er mwyn eu dadansoddi ymhellach. Ar dro, bydd yr elfen symbolaidd a syniadol yn rhy amlwg i'm bryd i, megis yn achos elfennau o Pridd neu Ta-ra Teresa. Ond pan fo’r cymeriad yn llefaru o ganol digwydd, fel sy’n digwydd i raddau helaeth yn Anweledig a 'Fel Stafell', dyna imi sy’n cynnig profiad theatrig grymus.

Cyfrwng arall i fynegi’r tensiynau rhwng dau fyd y dramodwyr yw eu defnydd o ddwy iaith swyddogol eu cenhedloedd diwladwriaeth. Fel Samuel Beckett y mae Belbel wedi dewis ysgrifennu yn ei ail iaith, sef Catalaneg. Yn Postcolonialism Revisited, disgrifia Kirsti Bohata y pellter hwn y mae ail iaith, neu iaith gyntaf nad yw’n llwyddo i gyfleu hanes a diwylliant y siaradwr, megis fel ‘a defining feature in postcolonial writing in a European language'.${ }^{99}$ Canlyniad hyn yn ôl Bohata yw bod yr awdur yn fwy parod i arbrofi a chreu hybridedd ieithyddol: cyfrwng arall ydyw i fynegi affasia diwylliannol. ${ }^{100}$ Mae’n hawdd gweld parodrwydd Belbel i arbrofi yn ei ddramâu a noda’r dramodydd ei hun fod y pellter y mae ei ail iaith yn ei gynnig iddo yn amlygu amwysedd iaith a 
gwelir yr awydd i ddatgymalu iaith mewn dramâu megis En companyia d'abisme, er enghraifft. ${ }^{101}$ Mae George hefyd yn pwysleisio’r modd y mae Belbel, yr ieithydd, yn llwyr ymwybodol o amwysedd iaith a dadleua George mor addas yn achos Belbel yw sylw Peter Conrad am Samuel Beckett, 'as he travels, he is constantly translating, aware that any word is a dubious, untrustworthy translation of a feeling' ${ }^{102}$

Er nad yw Williams yn ysgrifennu yn ei ail iaith y mae’r syniad yma o’r pellter y mae iaith yn ei gynnig iddo yn rhywbeth sy’n codi yn ymron pob un o’i ddramâu. Mae Williams yn olrhain yr ymdeimlad hwn yn ôl i nifer o ffactorau yn ei fywyd personol. Ni fwriedir darllen gwaith y dramodydd yng ngoleuni ei fywyd yma. Fodd bynnag, yn yr achos hwn teimlaf fod y ffactorau hyn o gymorth inni wrth ddeall yr hyn sy’n ei osod ar y cyrion ac yng nghanol y cysyniad o affasia diwylliannol. ${ }^{103}$ Esbonia Williams fod llais gwan ei Dad yn elfen ddylanwadol ar ei waith: 'fe roddodd llais fy Nhad i ar ochr, ar ymyl rhywbeth, ymyl gwan fel arfer, y moesol fel a'r fel, rhywun oedd yn dioddef mewn rhyw ffordd arbennig' ${ }^{104}$ Yn ogystal â hyn, mae ei gyfnodau yn byw dros y ffin yn Lloegr hefyd wedi rhoi'r persbectif hwn o bellter ieithyddol iddo. ${ }^{105}$ Dadleuwn hefyd i’w frwydr ag alcoholiaeth gael effaith debyg arno, rhywbeth arall sy'n ei alluogi i fynegi'r cyflwr o fod 'rhwng' gwahanol bethau. Yn wir, y mae Williams yn cyfeirio at hyn yn y gerdd 'C2 H5 OH':

Toddwr gwael fy neuoliaethau

Cyhydedd ffals fy mhegynau. ${ }^{106}$

Mewn cyfweliad gyda Manon Wyn Williams, meddai Williams: ‘[f]aswn i ddim yn dymuno alcoholiaeth ar fy ngelyn pennaf, tasai gen i un. Ond mae yna rywbeth yna hefyd sy’n fy ngosod i ar ymylon pethau.' ${ }^{107}$ Nid dweud y mae’r dramodydd mai alcohol sy’n hybu ei greadigrwydd; yn hytrach, y broses o oresgyn alcoholiaeth a'r profiad o edrych ar y byd o’r cyrion a chwrdd â phobl eraill sydd hefyd ar yr ymylon sy’n golygu fod ganddo rywbeth i’w ddweud a bod ganddo lais eto. ${ }^{108}$ O ran hynny, ‘[c]refu cymuned/ y poteli llawnion’ sy’n ei osod ar wahân. ${ }^{109}$ 
Gwelir y dramodydd yn cwestiynu’r berthynas rhwng ieithoedd yn, ‘Pryd Fuo Kathleen Ferrier Farw?'. Wrth ddatgan fod clefyd Alzheimer ar Mr Parry, gwna’r meddyg, sydd yn medru'r Gymraeg, hynny yn y Saesneg.

“Doctor,” me’ fi, “diolch i chi am ddeud hynna’n Susnag wrtha i. Mi fasach chi ’di bod yn rêl hen genna tasach chi 'di 'i ddeud o’n Gymraeg."

Mae'r Susnag yn dda i rwbath withia. 'N enwedig os ydach chi isio teimlo pelltar. A chadw hyd braich. ${ }^{110}$

Dyma ddieithrwch ail iaith y cymeriad yn lleddfu poen y newyddion drwg.

Er nad yw Belbel yn trafod y berthynas rhwng Sbaeneg a Chatalaneg mewn modd mor amlwg yn ei waith, ceir yn hytrach drafodaethau mwy cyffredinol sy’n deillio o’r berthynas hyd braich y mae ei ail iaith yn ei chynnig iddo. Er ei fod yn ysgrifennu yn ei ail iaith, mae honno’n ail iaith hyderus, ail iaith y mae rhai yn dadlau nad yw’n iaith leiafrifol bellach. ${ }^{111}$ Efallai mai dyna sy’n esbonio natur llai mewnblyg ymdriniaeth Belbel o berthynas y Gatalaneg a’r Sbaeneg yn ei ddramâu.

Llinyn amlwg sy’n amlygu cyswllt rhwng gwaith y ddau yw’r modd y maent yn ymserchu mewn iaith ac effaith andwyol yr ymserchu yna mewn rhywbeth nad oes modd ymddiried ynddo.

\section{Poen Realiti: themâu}

Ynghyd ag arbrofi gyda ffurf ac iaith, mae'r ddau hefyd yn dangos parodrwydd i fentro'n thematig gan gynnig dehongliadau ffres, llai poblogaidd, o bosibl, o hen themâu ynghyd ag amlygu rhai pynciau tabŵ anodd i ddelio â nhw, rhywbeth sydd wedi cymell Roger Owen i ddadlau 'mai'r elfen amlycaf sy’n awgrymu newydd-deb gwaith Williams yw aflonyddwch eithafol ei weledigaeth'. ${ }^{112}$

Gwelir yr awydd hwn i aflonyddu yn Forasters gan Belbel a 'Ta-ra Teresa’ gan Williams. Drama am berthynas Cymru â Lloegr ydyw ‘Ta-ra Teresa’. Nid drama wrth-seisnig ydyw, fel mae’n siŵr yr oedd nifer yn ei disgwyl. ${ }^{113}$ Gwelir yr awydd hwn i’n deffro o’n trwmgwsg yn aml yn nramâu 
Williams. Ystyrier y geiriau hyn o ‘Pêl Goch’, er enghraifft: ‘Mi fyddwch chi farw o’r felan toc yn hiraethu am Llywelyn. ${ }^{114}$ Yng ngeiriau cymeriad ‘Ta-ra Teresa’, Johnny Heneghan, mewnfudwr o Lerpwl: 'Wales I realised was full of hushed things. Like the sound of someone undressing. On their own. Late at night. On their own. In the dark. ${ }^{115}$ Rhoi llais i’r sibrydion hyn a wneir yn y ddrama. Heb os, y mae Wiliams yn ‘tynnu’r menig gwynion oddi ar ddwylo Cymraeg’ a ‘gwneud i bobl ymdeimlo â'r sgrech a’r isleisiau sydd yr ochr arall i’r gân yng Ngwlad y Gân' ${ }^{116}$ Gellid dweud bod hynny’n wir am Forasters hefyd. Cynnig persbectif o’r cyrion ar eu cenhedloedd a wna'r ddau yn hynny o beth. Efallai bod y persbectif a gynigiodd ei brofiad ei hun o symud i Gatalwnia o dde Sbaen wedi bod o gymorth i Belbel wrth gyfansoddi'r ddrama. Drama deuluol ydyw Forasters sy’n neidio rhwng dau gyfnod, sef y 1960au a dechrau’r unfed ganrif ar hugain. Mewnfudo a’r grymoedd dinistriol o fewn rhai teuluoedd sydd wrth galon y ddrama, fel sydd hefyd yn wir am 'Ta-ra Teresa'. Er mai canolbwynt Forasters yw un teulu Catalaneg, sy’n byw yn y fflat lawr llawr, ceir ynddi ddau deulu o fewnfudwyr hefyd, sef y Sbaenwyr o dde Sbaen yn y 1960au a’r teulu o Ogledd Affrica ar ddechrau'r unfed ganrif ar hugain. Trwy gyfosod a gwrthgyferbynnu’r dylanwadau 'estron’ hyn y datblygir gwrthdaro’r ddrama. Egyr y cyfan gyda’r bachgen (o’r 1960au), sydd bellach yn ddyn busnes llwyddiannus, yn prynu'r fflat oddi wrth fab y teulu Catalaneg a oedd yn byw o dan ei deulu am flynyddoedd. Byddai modd dehongli hyn fel symbol gobeithiol ar gyfer Catalwnia gyfoes, fwy goddefgar ac amlddiwylliannol. Mae gweddill golygfeydd y ddrama yn neidio rhwng cyfnodau amseryddol er mwyn cwblhau'r darlun o’r teulu dinistriol a'u hagweddau amheus tuag at eu cymdogion ‘estron’. Er bod Forasters, fel ‘Ta-ra Teresa', yn gorffen gyda marwolaeth, perthyn arwyddocâd symbolaidd ac elfen o obaith i'r ddwy farwolaeth. Yn achos Forasters, mae’n dynodi marwolaeth y chwaer a’r Fam, dau gymeriad sydd yn ôl David Geroge yn symbol o’r cancr a’r culni a oedd yn bodoli o fewn y teulu megis y fam Sbaenaidd ormesol yn Tŷ Bernada Alba gan Lorca. Yn achos 'Ta-ra Teresa', mae penderfyniad Robat Hefin i gyflawni hunanladdiad hefyd yn meddu ar arwyddocâd symbolaidd. Ac fel yn achos, Forasters, neidiwn rhwng y presennol a'r gorffennol yn y 
ddrama hon hefyd, ond y tro hwn, ar ffurf sgwrs radio gyda Beti George fel rhan o’i rhaglen boblogaidd Beti a'i Phobol. Trwy gyfrwng y sgwrs radio gwelwn fersiynau cyferbyniol o fagwraeth Robat Hefin, a gwelwn trwy hynny sut y mae Robat Hefin yn newid a mowldio'r gorffennol er mwyn creu delwedd fwy 'parchus' a 'derbyniol' ohono ef ei hun. Y mae ef, fel ei fam yn byw celwydd er mwyn hyrwyddo delwedd ddelfrydol o’r dosbarth canol Cymreig. Dysgwn, er enghraifft, nad y dyn mud di-asgwrn-cefn o Gymro yw ei dad mewn gwirionedd ond y Sais o Lerpwl, sy’n cael perthynas â’i fam, sy’n mynnu cysylltiadau â Thryweryn. ${ }^{117}$ Wrth iddo gyflawni hunanladdiad y mae’n dynodi diwedd y bywyd Cymreig dosbarth canol, ymddangosiadol barchus hwnnw y mae’n ceisio ei efelychu. Adrian, nid Robat Hefin, ydyw’r symbol o obaith yn y ddrama. Tystiwn i drawsnewidiad y Saesnes a dry’n Gymraes o’n blaenau ar y llwyfan. ${ }^{118}$ Wrth i Beti George ofyn ei chwestiwn olaf i Robat Hefin, sef pam y cymerodd y naid honno o flaen y car, naid nid annhebyg i un Dewi yn Cymru Fydd, y mae’n ateb trwy ddweud:

Oherwydd i mi weld, Beti. Gweld hogyn bach rochor arall i’r lôn ar y pafin yn sgrechian crio iddo fo'i hun. Ac ar 'i ysgwydd o. . . Bwswca. . . A hwnnw ddim yn gweithio. Ac wrth 'i ochor o ddynas a llond 'i haffla hi o bacia a rheiny'n disgyn i bob man fel petai hi ei hun yn dadfeilio. Ac yn sbïo ar y ddau. . .Y dyn 'ma. . .Fel dyn diarth. . .A'i geg o ar agor yn ogof ddu fel petai o'n mynd i ddeud rhwbath. Ond mi roeddach chi'n gwybod 'i fod o wedi colli 'i lais. . .Fod rhywun wedi rhigo'i focs llais o'i wddw fo. . .Ac nad oedd modd iddo fo ga'l hyd i'r geiria. . .Ac wrth sbïo arnyn nhw, ddirnad Beti, fod yn rhaid i rai petha farw. Nad oes dichon i chi fedru 'u hachub nhw. Eu bod hi'n amhosibl eu gwaredu nhw o'r gorffennol. . . ${ }^{119}$

Ceir yn y geiriau olaf hyn o enau Robat Hefin allwedd i’r symbolaeth, a gwelwn fod cymeriadau Williams, fel cymeriadau Belbel, yn byw trwy gyfnod o affasia diwylliannol, a phoen a gwacter y cyfnod hwnnw y mae Feldman yn cyfeirio ato. Symbol yw'r bagiau uchod o'r celwydd y mae mam Robat Hefin, y fam ddinistriol sy’n cwbl groes i’r ddelwedd fwy traddodiadol o’r Fam Gymreig, yn eu cludo er mwyn celu'r gwir a chyfleu bywyd parchus dosbarth canol Cymreig. Gwelwn hefyd y ffigwr tadol mud a di-asgwrn-cefn. Gyda marwolaeth Robat Hefin, fel sy’n wir am farwolaeth y fam/ferch yn Forasters, gwelwn ddiwedd byw'r celwydd parchus sydd ond yn arwain at ymdeimlad o dwyll a gwacter a rhagrith, at affasia diwylliannol: 
ROBAT HEFIN:. . .Isio newid, ia? Gyffesu i mi fy hun fy mod i wedi syrffedu ar Steddfod a Stomp a Thalwrn a Phrotestio. Fod y geiria Cymraeg yn ddrain yn 'y ngheg i. Isio llonydd, ia? Isio distawrwydd, ia? 'Laru tu mewn. Gwenu tu allan. Geiriau'n ffwndro tu mewn. Rhethreg tu allan. . .Ac nad oedd na chapal nac eglwys nac emyna na Beibil wedi golygu dim imi ers duw a ŵyr pryd. ${ }^{120}$

Gellid ystyried Forasters a 'Ta-ra Teresa’ yn enghreifftiau o Drydydd Gofod Bhabha. Dramâu ydynt sy’n pwysleisio hybrydedd y Gymru a Chatalwnia gyfoes sy’n ymwrthod â'r syniad o begynau cyferbyniol. Deisyfir y ‘newid’ y 'llonydd’ a’r ‘distawrwydd’ y mae Robat Hefin yn sôn amdano uchod, wrth i’r geiriau ‘ffwndro’. Ac ynghyd ag amau grym geiriau, mae’r ddau fel pe baent yn amau grym y Gair yn eu gwaith:

Y mae'r syniad o 'dduw' wedi newid rhwng 1990 a 2012: gŵyr pawb hynny ond yr Eglwys. Nid yw Darwin, Marx a Nietzsche, Freud, y Somme, Auschwitz, Hiroshima yn mennu dim ar feddylfryd yr Eglwys yng Nghymru. A hynny yw'r peth, debygaf fi: fod y posibilrwydd o 'Dduw Hollalluog' wedi erydu gymaint yn ein plith yn y gymdeithas Orllewinol gyda threigl amser ac am nifer fawr o resymau - rhai o’r rhesymau hynny ymhlith yr enwau yn y frawddeg flaenorol - er, efallai, nad ydym yn gwbl ymwybodol pa mor bell y mae'r erydu wedi ei gyrraedd. ${ }^{121}$

Dyma ymwybod o bellter, ond ni ŵyr y dramodydd 'pa mor bell' y mae'r genedl wedi symud oddi wrth ei gorffennol crefyddol. Mewn modd tebyg, er bod dau gymeriad En companyia d'abisme, Belbel yn medru gwerthfawrogi bod i iaith grefyddol elfen o brydferthwch, nid oes i'r iaith honno ryw lawer o rym yn eu llygaid hwy ychwaith:

YOUNGER MAN: I don’t think that consciously reproducing phrases is exactly intelligent.

MAN: Ah, here we go again, then?

YOUNGER MAN: Where?

MAN: “I-don't-think-learning-and-trusting-are-the-same-thing,” "I-don't-think-thatconsciously-reproducing-phrases-is-exactly-intelligent”. . .Huh! You should take up writing religious statue books, manuals or catechisms [. . .]

YOUNGER MAN: Yes, sir, if one day I do write a manual or a catechism, I'll ask your help. So at least the vocabulary will be rich and varied, even if it has no real substance! ${ }^{122}$

Dyma’n rhannol pam y mae dramâu Williams a Belbel yn apelio’n syniadol heddiw. Mae’r dramodydd yn barod i godi cwestiynau sensitif sy’n berthnasol i Ewrop seciwlar sy’n parhau’n Gristnogol ac yn ôl-Gristnogol ar yr un pryd. Cyflwynir inni’r cwestiynau am y ddelwedd ddeublyg a geir o Dduw, Duw’r Beibl a’r Duw sy’n caniatáu’r holl erchyllterau y cyfeirir atynt uchod. Yn 2015, 
darlledwyd cyfres o'r enw Dal i Gredu? ar S4C, a bu'r ymateb yn frwd wrth iddi archwilio'r modd y mae Cymru mwy seciwlar, ôl-grefyddol yn dal i arddel rhyw fath o gred annelwig mewn Duw. ${ }^{123} \mathrm{~A}$ dyfynnu Williams, yr offeiriad, a’r mab i offeiriad: ‘[r]wy’n byw mewn amwysedd crefyddol. Ond nid wyf yn gollwng fy ngafael. ${ }^{, 124}$ Gweler enghraifft o’r amwysedd hwn isod yn 'Pêl Goch':

Fy Nuw!. . Fy Nuw! . . Fy Nuw! . . Dwi ’di colli'r iaith . . . Ma’r geiria odd gin i’n arfer bod 'di pydru ... Ma' nhw hyd lawr fy enaid fel fala drwg ... Fedra i 'im ond teimlo'r pelldar oddi wrthyn nhw ... A'r oerni . . . A'r dim byd. ${ }^{125}$

Dyma’r 'pelldar' sy’n nodwedd ar affasia diwylliannol, sy’n deillio o'r profiad hwnno o fod rhwng, rhwng ffydd ac anffyddiaeth a’r ymdeimlad bod un wedi anghofio’r stori a oedd yn rhan annatod o fywyd cynifer am gyhyd. Fel y nododd Nic Ros:

Mae crefydd wedi dirywio gymaint yng Nghymru ac yn y byd gorllewinol, ond mae o'n fwy nodedig yng Nghymru nag yn yr un lle arall dwi'n meddwl, nes wyt ti wedi colli hanfod crefydd, sef i mi canfod storïau a delweddau sydd yn gwneud synnwyr o fywydau pobol. ${ }^{126}$

Gellid dadlau yr un peth am waith Belbel. Ymhyfryda yntau yn yr amwysedd rhwng dau fyd, rhwng cred ac anffyddiaeth. Er nad yw’r gyfeiriadaeth grefyddol a’r ymdriniaeth o grefydd yn bresennol ym mhob un o’i ddramâu y mae’n britho nifer ohonynt megis, En companyia d'abisme a drafodwyd eisoes a Morir:

LLOFRUDD: Ca’ dy ben. Gad i fi weld. . .Dwedodd e’ wrtha i am beidio â'i ladd. . .Paid â’i ladd er mwyn ... Duw. Er mwyn ... Duw ... Duw? Iawn, dyma'r her: galw di ar Dduw a gofyn iddo ddod i’w achub. Iawn? Achos ti’n credu ynddo fe, nag yw ti?

DIODDEFWR: Ydw. A mi wyt ti yn hefyd.

LLOFRUDD: Fi? Sa i’n shwr. Drych, os ydy fe'n ymddangos mewn pum munud gan fy stopio i rhag ei ladd e', fe greda' i ynddo fe. Wrth gwrs y gwna' i. Nawr te, gad i ni ddechrau. ${ }^{127}$

Nid yw’r naill ddramodydd na’r llall yn ystyried eu hunain yn ddramodwyr gwleidyddol. Yn wir, y mae Williams wedi dadlau mai’r ffaith nad yw erioed wedi teimlo ymdeimlo’n genedlaetholwr sy’n golygu na fedrodd fynd dan groen cymeriad Robat Hefin yn 'Ta-ra Teresa', cymeriad a ddisgrifiwyd gan Williams fel ei gymeriad gwannaf. ${ }^{128}$ Nododd Xavier Puchades y byddai geiriau Koltès -“I have 
never had political ideas in my plays”- yn addas yn achos Belbel, eto nid yw ei waith, fel gwaith Koltès ei hun yn swilio rhag problemau cymdeithasol mawr. ${ }^{129}$ Mae’n annatod bod elfennau gwleidyddol yn treiddio i waith y ddau sy’n ysgrifennu mewn cenhedloedd diwladwriaeth. Fel yr awgrymodd Saunders Lewis yn ‘Egwyddorion Cenedlaetholdeb’, gellid dadlau bod y weithred o ysgrifennu'n Gatalaneg ac yn Gymraeg yn weithred wleidyddol ynddi ei hun. ${ }^{130}$ Er bod Belbel yn cyfieithu nifer o’i ddramâu i’r Sbaeneg, nid yw’n gwneud hyn er mwyn eu hanfon at y Centro Dramatico Nacional (sef Theatr Genedlaethol Sbaen), eithr fel yr esboniodd:

My plays are more welcomed in Germany than in Madrid, and that might be due to political reasons. I've just finished a play [Forasters (2004)] and I am translating it into Castilian in order to send it to Germany, France, and Sweden, not to Madrid. ${ }^{131}$

Efallai mai’r themâu affasig yma sy’n ddieithr i gynulleidfaoedd Madrid a’m denodd i at waith Belbel i ddechrau, a'r ffaith ei fod ef fel Williams yn amlygu'r profiad o fod rhwng dau fyd. Dramodwyr y cyrion sy’n ganolog i’w diwylliant ydynt. ${ }^{132}$ Mae’r pwt isod o La Sang gan Belbel yn esiampl o hynny. Anodd peidio â meddwl nad oes yma ddramodydd gwleidyddol:

CHILD: I've learned about hating the enemy ever since I was born. (Pause.) It's not easy to get rid of hate when you're grown up with it. (Pause.) And with all the harm the enemy keeps causing us every day $[. . .]^{133}$

Cynnig y ddau ddramodydd hefyd gip ar abswrdiaeth ein bywydau, megis yn 'Ta-ra Teresa’ a ‘Chwilys’ gan Williams neu La Sang gan Belbel. Yr hyn a wna La Sang ydyw pwysleisio mor absẃrd ydyw ceisio byw bywyd 'normal' o ddydd i ddydd tra bod erchyllterau yn digwydd yn y byd o’n cwmpas. Drama a luniodd mewn ymateb i lofruddiaeth Miguel Angel Blanco gan ETA yn 1997 ydyw ac mae La Sang yn enghraifft o ddrama arall sy’n dangos y tensiynau rhwng dau fyd gan fynegi abswrdiaeth bywyd yn wyneb erchyllterau o'r fath. ${ }^{134}$ Er mai cyfeirio at un o'r erchyllterau a gododd yn sgil grŵp terfysgol yng Ngwlad y Basg a wna yn La Sang, nid yw’r ddrama hon, fel sy’n wir am nifer o ddramâu Belbel a Williams fel ei gilydd, wedi’i gwreiddio yng Nghatalwnia na Chymru. Yn wir, mae Belbel yn gwneud ymdrech ymwybodol i osgoi hyn, oherwydd yn y cyfnod ôl-Franco gwelwyd ymgais ymwybodol i ryngwladoli Catalwnia, gan wleidyddion ac ymarferwyr 
theatr fel ei gilydd. Wrth reswm, anodd osgoi'r paradocs mewn perthynas â'r cyfryw ryngwladoli. A dyfynnu Feldman:

At the same time, the city, paradoxically, began to take on a nearly invisible, ghostly presence on the contemporary stage. Curiously, in the outpouring of Catalan plays written during the post-Franco period, Barcelona and/or Catalunya are, in general terms, conspicuously absent from the theatrical landscape. In the private, intimate space of theatrical writing, the dramatists themselves, especially throughout the decades of the 1980s and 1990s, appeared to elude for the most part any sort of cultural specifity. ${ }^{135}$

Roedd fel pe baent yn meithrin hyder eto ar ôl cyfnod hir o autoodi (o hunan gasineb), a olygai’n aml eu bod am osgoi enwi trefi a dinasoedd Catalwnia. ${ }^{136}$ Yn hyn o beth, bod rhwng dau fyd, rhwng Catalwnia cyfnod Franco a Chatalwnia hyderus sydd am fod yn annibynnol a yrrodd dramodwyr i beidio â lleoli eu dramâu yn amlwg yn Nghatalwnia ac yn agored i nifer o gynulleidfaoedd. Yn wir, mae David George wedi nodi pwysigrwydd yr ymateb rhyngwladol a fu i waith Belbel. ${ }^{137}$ Mae Helena Buffery hefyd wedi amlygu'r cyswllt rhyngddo â gwreiddioldeb:

In the case of Catalan theatre and culture, as is liable to be the case in any minoritised, dependent or (post)colonial culture, the perceived need and demand for originality is in many ways stronger than ever, internally and externally, as a pre-requisite for visibility. ${ }^{138}$

Roedd bod yn fentrus trwy wthio ffiniau theatrig, tra’n osgoi cyfeirio at leoliadau penodol, yn gyfrwng i’r theatr Gatalaneg serennu’n rhyngwladol, yn hytrach na pharhau i fyw dan gysgod Sbaen. ${ }^{139} \mathrm{Ar}$ un llaw gellir beirniadu’r awydd i lefaru ag eraill yn rhyngwladol a pheidio â lleoli’r ddrama Gatalanaidd yn nhir a daear Catalwnia ei hun. Anodd peidio â meddwl am feirniadaeth enwog Gerallt Lloyd Owen wrth iddo gyfeirio at awydd rhai Cymry i droi yn 'eithafol ryngwladol wŷr'. ${ }^{140}$ Ac eto, cofiwn i Saunders Lewis herio’r Cymry drwy gyfrwng stori Esther a Brad a sut y dewisodd Gwenlyn Parry dŵr amwys a thŷ mewn lleoliad amhenodol ar y tywod yn gyfrwng i herio cynulleidfaoedd y chwedegau. Gall yr annelwig a’r rhyngwladol fod yn drosiad addas ar gyfer herio'r gynulleidfa leol ac ennill cydnabyddiaeth rhyngwladol ar yr un pryd. Dramodydd digon annelwig yw Williams. Ystyrier y disgrifiad hwn o set Pridd: 
Tybiaf na ddylid cael set naturiolaidd oherwydd - efallai - mai ym mhen Handi Al yn unig y mae hyn i gyd yn digwydd, os y digwyddodd o gwbl[. . .] Daw clown i mewn i'w 'ystafell fyw' yn ei 'gartref'. (Nid yw'n tynnu na'i wisg na'i golur drwy gydol y perfformiad, gyda llaw.) Ar y 'dodrefn', $y$ 'cadeiriau' ac ati mae pridd a thywyllwch. (Byddai'n wych, wrth gwrs, cael 'dodrefn' wedi eu gwneud allan o bridd, a'u bod mewn 'cae' - neu awgrym o 'fynwent'. ')

Mae’r lleoliad hwn yn swnio’n debyg i ddisgrifiad J. Sanchis Sinisterra o set Belbel;

‘[mewn] lle tywyll nad yw’n ceisio efelychu unrhyw beth, nad yw’n deisyfu cynnwys unrhywbeth ac eithrio’r hyn ydyw: y senario’. ${ }^{142}$ Fel ei gymeriadau, cyfrwng yw’r set i archwilio syniad. Gwelir hyn yn Merched Eira hefyd, drama a leolir mewn lle amhenodol yng nghanol llawer o eira. Gwelir yma eto Williams yn archwilio un o hoff themâu Belbel, amser a'r cof, ynghyd â marwolaeth. Caiff y gair ei ailadrodd er mwyn pwysleisio hynny:

Edna: . . .Amser (yn oer)

Edith: Dyna i ti ddiawl o beth i’w adael ar ôl mewn Hôm. . .Amser i feddwl. . .Amser i sbario. . .Amser i bendroni. . .Amser i gofio. . .

Edna: Na! Nid amser i ddim byd. Jyst amser. Amser ar ei ben i hun, noeth. . .Tician. . . Tician. . .Tician. . . 143

Fel yn achos cymeriadau Diwéddgan gan Samuel Beckett a 'Wal' gan Williams, mae Edith ac Edna yn dibynnu ar ei gilydd. Mae Belbel hefyd yn hoff o archwilio natur lithrig amser, fel y nododd Enric Gallen mewn perthynas â A la Toscana. A gwelir y thema hon hefyd yn Dins la Seva Memòria. ${ }^{144}$
3: Nawr
1: cofia'r gorffennol yn dy gof
2: mae'r foment wedi dod.
3: Hon yw'r foment.
1: Pan oedd yr amser hwn
2: wedi ei barlysu eisoes
3: a dy feddiannu .
2: Cymaint o ddiwrnodau!
3: Sut gymaint o ddiwrnodau!
1: Fedri di eu cyfrif.
2: Cymaint.
3: Sut gymaint o ddiwrnodau. ${ }^{145}$

Gwelir y modd y mae'r cymeriadau yn archwilio annifyrrwch treigl amser uchod. Gellid dadlau mai annifyrru a wna Williams a Belbel yn fynych yn eu dramâu felly trwy godi cwestiynau mawr eu cenhedloedd, sydd erbyn hyn yn y cyfnod ôl-Franco yng Nghatalwnia, ac ers datganoli yng Nghymru 
yn genhedloedd mwy hyderus. Yn wahanol i rwystredigaeth Dyn 1 yn Chwilys Williams isod, nid cuddio a wna'r ddau ddramodydd hyn:

Dyn 1:. . Cuddio mae paent Kyffin nid datguddio. Peintio dros rwbath mae o o hyd. A merwino'n hunan-ddealltwriaeth ni hefo'i liwiau syber, hollol ddisgwyliadwy. Yr un un lle ydy Cymru ar ôl i Kyffin edrych arni. A dyna pam maen nhw'n crogi ar furiau crand y dosbarth canol. Badj ar y wal ydy Kyffin. Nid llun. Fedraim fforddio Kyffin mewn mwy nag un ystyr. ${ }^{146}$

Os ‘cuddio’ y mae paent Kyffin, crafu’r paent i ffwrdd y mae’r dramodydd. Chwilio am yr hyn sydd rhwng y cynfas a’r llun arwynebol.

\section{$\underline{\text { Clo }}$}

Rhoddwyd y teitl 'Dramodwyr rhwng dau fyd' ar yr ysgrif hon a cheisiwyd dangos sut y mae Williams a Belbel yn llwyddo i fynegi'r profiad hwnnw o affasia diwylliannol, y profiad sy’n fynegiant o fod 'rhwng' dau beth , rhwng natur symbolaidd Theatr yr Absẃrd a dychwelyd yn glasurol at y testun tra'n parhau i amlygu aneffeithlonrwydd iaith yn aml, rhwng syniad gwyrdroëdig eu cenhedloedd o realiti a diffiniad moel a brawychus y dramodwyr o'r hyn yw realiti. ${ }^{147}$ Yn y tyndra o fod 'rhwng' dau beth, rhwng barddoniaeth a rhegfeydd, ac ar adegau barddoniaeth y rhegfeydd, yr amlygir cwestiynau mawr eu cenhedloedd; rhwng gadael Duw a pheidio â’i adael yn llwyr, rhwng gadael Lloegr i raddau a glynu wrthi fwyfwy yn wyneb Brexit. Dyna gamp y ddau ddramodydd hyn. Ac er yr holl gwestiynau, ni chynigir ateb; ‘[m]arciau cwestiwn yn atseinio ym mudandod mawr yr awditoriwm' yw'r cwestiynau a leisir yn eu dramâu. ${ }^{148}$ Nid yw’r profiad o ‘affasia diwylliannol’, sy’n deillio o’r profiad o fod 'rhwng', ennill a cholli, ddoe ac yfory, yn unigryw i Gymru a Chatalwnia ychwaith. Ceir adleisiau ohono yn theatr Galisia ac Iwerddon hefyd, cenhedloedd eraill sy’n ymgodymu â hunaniaeth ddeuol a thensiynau diwylliannol.

Ar ddechrau'r ysgrif, dyfynnais gwestiwn Cathy Lenny mewn perthynas â'r theatr yn Iwerddon wrth iddi ofyn sut yr oedd y theatr gyfoes yn mynd i archwilio’r cyflwr hwn o fod rhwng gwahanol 
fydoedd, cyflwr yr oedd hi’n cyfeirio ato fel 'inbetween-ness’. Er na cheisir ateb y cwestiwn hwnnw yng nghyd-destun y theatr yn Iwerddon yma, awgrymir bod gwaith Belbel a Jones Williams yn llwyddo i ymateb i’r her honno yng Nghymru a Chatalwnia gan apelio’n syniadol-theatrig at eu cynulleidfaoedd. Y mae geiriau’r cymeriad Jiwdas o ddrama Williams Iesu!, yn llwyddo i fynegi camp y ddau awdur i’m bryd i:

Siarad i gyflwr y bobl mae hi, nid i’w crebwyll nhw [. . .] Nid diffinio'r pethau y mae ei geiriau hi ond consurio cymdeithas amgen [. . . ] ac mae'r bobl yn ymateb hefo'u hemosiynau a'u breuddwydion. Ac onid dyna beth y mae gwleidydd eisiau - emosiwn a breuddwydion pobl, eu symud nhw o'r ffeithiau i fyd arall. ${ }^{149}$

Eu hawydd i siarad â chyflwr pobl sy’n byw mewn ‘cymdeithas amgen’, sef y cyflwr hwnnw o fod yn y Trydydd Gofod rhwng dau fyd, sy’n golygu bod Williams a Belbel fel ei gilydd yn cael eu hystyried yn ddramodwyr blaenaf eu cenhedloedd a'u cenhedlaeth.

Yn ei thraethawd doethurol y mae Manon Wyn Williams yn dadlau nad oes modd cyfieithu gwaith Williams:

Y mae cryfder presenoldeb y thema o Gymreictod yn nifer o ddramâu Aled Jones Williams yn golygu y byddai agweddau hollbwysig o'r gweithiau hynny yn cael eu colli wrth eu cyfieithu gan eu bod yn trafod sefyllfaoedd sy'n berthnasol i Gymru ac i’r Cymry Cymraeg eu hiaith yn arbennig [. . . . $]^{150}$

Deallaf, wrth gwrs, y ddadl parthed anghyfieithiadwyedd y tafodieithol a'r lleol. Yn wir, meddai Williams ei hun:

Maen nhw'n Gymraeg yn eu hiaith. Dwi ddim yn meddwl fedri di gyfieithu lot ohonyn nhw, oherwydd mae'r idiom Gymraeg yna, a ffordd o ddeud rhywbeth sydd o’i drosi i iaith arall yn mynd ar goll. A mae fy naearyddiaeth i’n gysáct iawn. Rhyw ddwy filltir sgwâr ydy o, a mae cerflun Lloyd George [ar y sgwâr yng Nghaernarfon] yn y canol. Felly ym mha ystyr maen nhw'n Gymraeg? Wel maen nhw'n dwad yn sicr o Gymru, o'r diriogaeth yna, ac yn deud pethau gwahanol i beth sydd wedi cael ei ddeud. ${ }^{151}$

Fodd bynnag, perthyn elfen o ansicrwydd i eiriau'r dramodydd uchod, 'marc cwestiwn fel bach yn hongian o wialen [ei] eiriau’, a gobeithiaf i'r ysgrif hon ddangos nad yw'r profiadau a leisir gan Williams mor gysáct eu daearyddiaeth ag y mae’r dramodydd ei hun yn ei dybio mewn 
gwirionedd. ${ }^{152}$ Yn ei gyfrol o gerddi, Y Cylchoedd Perffaith, ceir cerdd sy’n dwyn y teitl '?'. Dyma bennill olaf y gerdd honno:

Crist y Cyfarwydd!

Godrapia! Os medrwch chi'i wisgo fo

mewn dillad Ymerawdwr

a'i alw'n Pantocrator

neu ei ddilladu'n fataffisegol

â berfau Groeg

mi fedrwch ei Gymreigio

yn Wydion Iesu

y gorau cyfarwydd yn y byd oedd.

Yn yr un modd ag y mae Williams yn barod i wthio’r ffiniau a chorddi’r dyfroedd gyda’i ddelwedd o Iesu, drama sy’n seiliedig ar Efengyl Marc, ond sy’n cynnig ei fersiwn ei hun o’r Efengyl honno mewn gwirionedd, yma, dadleuaf o blaid rhannu’r llais hwnnw ag eraill. ${ }^{153}$ Yn yr ysgrif hon cyfieithais bytiau o ddramâu Belbel i’r Gymraeg a chyfeirio at gyfieithiadau Saesneg o’i waith gan ddangos bod nifer o brofiadau rhwng y ddwy genedl yn gyffredin ac yn berthnasol i’w gilydd gan adleisio’r hyn y mae R. M. Jones yn ei ddadlau am genhedloedd ôl-drefedigaethol: ‘mae ganddyn nhw rywbeth i’w ddweud wrth y ddynoliaeth yn ei gwendid sy'n wahanol i’r hyn sydd i'w ddweud gan y trefedigaethwyr.' ${ }^{154}$ Mi fyddai cyfieithu'r lleisiau hynny yn fodd o ochel rhag culni o fewn ein theatr, '[i]n terms of dramatic fictions the real prison of a colonial, or quasi-colonial, situation is the sheer narrowness of the range of definitons of 'being Welsh' that have been made available.'155 I'r perwyl hwn, adleisia’r beirniad theatr Ed Thomas y dramodydd:

I want a Wales at ease with itself and rejoicing in its natural eclecticism. I don't want to see Wales locked in a debate about Welsh and English. I want a multicultural Wales with a myriad sustainable myths [...] The new Wales has to be fast, maverick and imaginative, and innovative and inventive in its aim to be a small, interesting country within a European context, a country where the albatross of Britain has finally fallen from its neck. It certainly has fallen from my neck. I like England, but not Britain. ${ }^{156}$

Ymddengys fod nifer yn teimlo fod y theatr yng Nghymru yn methu’n llwyr â gwneud hyn ar hyn o bryd. Ystyrier sylwadau grŵp o 40 o ddramodwyr Cymru mewn llythyr agored at National Theatre Wales yn ddiweddar yn lleisio eu hanfodlonrwydd ynghyd â sylwadau Ian Rowlands a Paul Griffiths 
am y Theatr Genedlaethol yng Nghymru. ${ }^{157}$ Rhaid i ni ehangu ein gorwelion ac ymgysylltu ag eraill a ddaliwyd rhwng eu darostyngiad a'u rhyddid, eu cymhathiad a'u harwahanrwydd; codi drychau perthnasol, a defnyddio trosiad Josep-Anton Fernàndez. ${ }^{158}$ Dadleua ef mai diffyg drychau effeithiol yn y theatr oedd y broblem yn achos Catalwnia. Hynny yw, diffyg drychau sy’n dangos pobl, problemau a heriau’r wlad. Dyna, ym marn Fernàndez, a oedd yn golygu bod Catalaniaid yn dechrau teimlo math o ddieithrwch a hunan gasineb, yr autoodi hwnnw y cyfeiriwyd ato eisoes, a mabwysiadu’r ddelwedd anffafriol honno a dafluniwyd ohonyn nhw eu hunain, oherwydd yr unig ffordd yr oedden nhw'n gweld eu hunain oedd trwy’r drych o’u blaenau. Y drych gwyrdroëdig yn llaw’r trefedigaethwyr nad oedd yn gweddu iddyn nhw. ${ }^{159}$ Mynega Ian Brown ddadl debyg mewn perthynas â phwysigrwydd cyfieithu dramâu Quebecois Michael Tremblay i’r Saesneg a’u llwyfannu yn yr Alban, 'Tremblay was writing out of another national community within a larger political entity, with its own language and identity issues. The experience of Scotland was clearly not

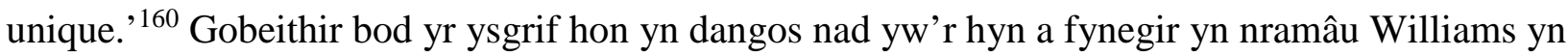
anghyfieithiadwy ychwaith a bod mawr angen rhagor o waith cymharol rhwng dramodwyr a chenhedloedd eraill sydd 'rhwng dau fyd' er mwyn profi geirwiredd sylw Alun Lewis; '[t]he world is much larger than just England, isn’t it? It'll never be just English or Welsh again’. ${ }^{161}$

Abertawe

HANNAH SAMS

${ }^{1}$ Carwn gydnabod i'r ymchwil gael ei noddi gan Ysgoloriaeth Goffa Saunders Lewis ac i’r ysgoloriaeth honno a nawdd gan Erasmus ganiatáu imi dreulio cyfnod yng Nghatalwnia yn dysgu'r iaith. Diolch hefyd i’r Athro David George am sgyrsiau ar waith Belbel ac i’r Athro Tudur Hallam am ei anogaeth a'i awgrymiadau gwerthfawr wrth baratoi'r ysgrif hon.

${ }^{2}$ R. M. Jones, Ysbryd y Cwlwm (Caerdydd: Gwasg Prifysgol Cymru, 1998), t. 5. 
${ }^{3}$ Er enghraifft: Catrin Wyn Edwards, 'Language-in-Education Policies, Immigration and Social Cohesion in Catalonia: The Case of Vic', International Journal of Bilingual Education and Bilingualism, 19, (2015), 530-545 ac Elin Royles, 'Llywodraeth ranbarthol a chymdeithas sifil yng Nghymru a Chatalwnia’, Gwerddon, 5, (2010), 27-52. Mae llyfr Paul Birt yn esiampl o lyfr sy’n edrych tua Chatalwnia ym maes barddiaeth Gymraeg; Paul Birt, Cerddi Alltudiaeth: Thema yn Llenyddiaethau Québec, Catalunya a Chymru (Caerdydd: Gwasg Prifysgol Cymru, 1997) ${ }^{4}$ Ystyrier er enghraifft: Anhysbys, 'Un Brilliante Belbel’, El Periódico, 26 November 1993, 2. Ac yn achos Aled Jones Williams: Roger Owen, 'Y Dieithredig', Barn, 522/523 (Gorffennaf/Awst 2006), 91.

${ }^{5}$ Sharon G. Feldman, In the Eye of the Storm (Lewisburg: Bucknell University Press, 2009), t. 166.

${ }^{6}$ Aled Jones Williams, Tuchan o Flaen Duw (Llanrwst: Gwasg Carreg Gwalch, 2012), t. 70.

${ }^{7}$ Feldman, In the Eye of the Storm, t. 166.

${ }^{8}$ Huw Roberts, 'Dramodydd yr Isfyd’ Barn, 410 (Mawrth 1997), 27-9.

${ }^{9}$ Feldman, In the Eye of the Storm, t. 167.

${ }^{10}$ Geiriadur Prifysgol Cymru, [Ar lein]. Ar gael: http://geiriadur.ac.uk/gpc/gpc.html, cyrchwyd 19 Medi 2018

${ }^{11}$ Ann Laura Stoler, Colonial Aphasia: Race and Disabled Histories in France’, Public Culture, 23, (2011), 125.

12 Sharon G. Feldman, 'Post-Franco theatre, yn David T. Giles (gol.), The Cambridge History of Spanish Literature (Cambridge: Cambridge University Press, 2004), t. 737.

${ }^{13}$ Cathy Lenny, 'Location, Location, Location: Plays and Realities: Living Between the Pre-modern and the Postmodern in Irish Theatre', yn Steve Blandford (gol.), Theatre \& Performance in Small Nations (Briston: Intellect, 2013), t. 23.

${ }^{14}$ Aled Jones Williams, ‘Awelon', Y Cylchoedd Perffaith (Caernarfon: Gwasg y Bwthyn, 2010), t. 19. 
${ }^{15}$ Homi Bhaba, The Location of Culture (London: Routledge, 1994), t. 38.

${ }^{16}$ Angharad George, ‘Trafferth mewn Tafarn a’r Gofod Hybrid’, Ysgrifau Beirniadol XXXI, 96-7.

${ }^{17}$ Bill Ashcroft. Gareth Griffiths, Helen Tiffin, Key Concepts in Post-Colonial Studies (London: Routledge, 1998), t. 118.

${ }^{18}$ Peter Childs a R. J. Patrick Williams, An Introduction to Post-Colonial Theory (London:

Longman, 1997), t. 134. 'hybridity shifts power, questions discursive authority and suggests, contrary to the implication of Said's concept of Orientalism, that colonial discourse is never wholly in the control of the colonizer'

${ }^{19}$ Aled Jones Williams, Oerfel Gaeaf Duw (Caernarfon: Gwasg Pantycelyn, 2002), t. 16.

${ }^{20}$ Ystyrier hefyd eiriau Nic Ros, 'Rhwng Rheg a Rhethreg', yn Nic Ros (gol.), Disgwyl y Býs yn Stafell Mam (Caernarfon: Gwasg y Bwthyn, 2006), t. 19: 'Methiant a cholled yw’r norm yng ngwaith Aled Jones Williams. Mae ei waith yn ddirfodol gan ei fod yn gyson yn archwilio'r posibiliadau ysbrydol mewn byd di-Dduw.’ A David George a John London, ‘Avant-Garde Drama’, Contemporary Catalan Theatre: An Introduction (Wiltshire: Cromwell Press, 1996), t. 73. Mae sylw George a London hefyd yn fy atgoffa o ddisgrifiad Janice Jones o Aled Jones Williams fel llenor sy’n 'gwirioni gydag iaith, ac yna, ar yr un gwynt bron iawn, yn ymwybodol o’i hannigonolrwydd.', Janice Jones, ‘Adolygiad - Aled Jones Williams, Y Cylchoedd Perffaith’, [Ar lein]. Ar gael: http://www.bbc.co.uk/cymru/cylchgrawn/llyfrau/adolygiadau/cylchoedd-perffaith.shtml , cyrchwyd 30 Hydref 2018.

21 "El teatre catala es diu Sergi Belbel". Avui, 4 January 1989, 4. [fy nghyfieithiad]

${ }^{22}$ Gweler rhestr gyflawn o’i wobrau yn Sergi Belbel, A la Toscana (Barcelona: Proa, 2007), tt. 11113.

${ }^{23}$ Roger Owen, 'Y Dieithredig’, Barn, 522/523 (Gorffennaf/Awst 2006), 91.

${ }^{24}$ Steve Blandford, 'Theatre and Performance in a Devolved Wales', Theatre \& Performance in Small Nations, tt. 65-6. Yn yr ysgrif hon, awgryma Blandford fod Iesu! ymhlith cynyrchiadau 
pwysicaf a mwyaf llwyddiannus Theatr Genedlaethol Cymru; 'In Iesu! there is a case for saying that Theatr Genedlaethol Cymru demonstrated the potential for a national theatre in the Welsh language to fulfil Rowlands’ dream, but that it has yet to do this with any level of consistency.'

${ }^{25}$ Huw Roberts, Carys Ll. Edwards, Graham Laker, 'Drama lwyfan o leiaf un awr o hyd', yn J. Elwyn Hughes (gol.), Eisteddfod Genedlaethol Frenhinol Cymru Bro Colwyn 1995: Cyfansoddiadau a Beirniadaethau (Llandybïe: Gwasg Dinefwr, 1995), tt. 20-1.

${ }^{26}$ Roberts, Edwards, Laker, 'Drama lwyfan o leiaf un awr o hyd', Eisteddfod Genedlaethol Frenhinol Cymru Bro Colwyn 1995: Cyfansoddiadau a Beirniadaethau, tt. 20-1. Nid beirniaid y gystadleuaeth yn 1995 yw’r unig feirniaid i esbonio addasrwydd y gair 'rhwng' mewn perthynas â gwaith Williams, ystyrier sylwadau Meinir Eluned Jones, ‘Dy Daith dy Hun’, Barn, 482 (Mawrth 2003), 49. ; 'Un gair y dylid ei briodoli i waith Aled Jones Williams yw 'rhwng’. Mae’r ffin yn denau rhwng y pegynau paradocsaidd, nid yw’r naill begwn yn cael y gorau ar y llall. Rhyngddynt mae bodlonrwydd arwynebol i’w ganfod, ac yno, yn rhywle, mae’r rhan fwyaf ohonom ni yn trigo, ond llwydda'r artist hwn i gyfleu’r ffrithiant sy’n digwydd pan ddaw'r pegynau i gyffwrdd â'i gilydd ac i grafu yn erbyn ei gilydd.’

${ }^{27}$ Owen, 'Y Dieithredig’ Barn, 92-3 a Roberts, Edwards, Laker, 'Drama lwyfan o leiaf un awr o hyd’, Eisteddfod Genedlaethol Frenhinol Cymru Bro Colwyn 1995: Cyfansoddiadau a Beirniadaethau, tt. 20-1.

28 Joan de Sagarra, “Un Feliz Encuentro”, La Vanguardia, September 19, 2004.

${ }^{29}$ Feldman, In the Eye of the Storm, t. 10.

${ }^{30}$ Edward W. Said, Culture and Imperialism (London: Chatto and Windus, 1993), t. viii.

${ }^{31}$ Bhaba, The Location of Culture, t. 211.

32 Jones, Ysbryd y Cwlwm, t. 5.

33 Ibid., t. 38.

${ }^{34}$ Feldman, In the Eye of the Storm, t. 10. 
${ }^{35}$ David Adams, Stage Welsh: Nation, Nationalism and Theatre: The Search for Cultural Identity (Llandysul: Gomer, 1996), t. 5.

${ }^{36}$ Daeth dramâu abswrdaidd i’r brif bob blwyddyn namyn un yn ystod y cyfnod byr hwn. Trafodir hyn yn fwy manwl yn Hannah Sams, 'Ffarwel i’r Absẃrd?: Agweddau ar y Ddrama Gymraeg Gyfoes, PhD anghyhoeddedig, Prifysgol Abertawe (2017), tt. 16-38.

${ }^{37}$ John Gwilym Jones a William R. Lewis, ‘Drama Hir’, yn T. M. Bassett (gol.), Eisteddfod Genedlaethol Frenhinol Cymru Maldwyn a'r Cyffiniau 1981: Cyfansoddiadau a Beirniadaethau, (Llandysul: Gomer, 1981), t. 150.

${ }^{38}$ Bethan Jones a Siân Summers, 'Drama Hir Agored’, yn J. Elwyn Hughes (gol.), Eisteddfod Genedlaethol Cymru Tyddewi, Sir Benfro 2002: Cyfansoddiadau a Beirniadaethau, (Llandybïe: Gwasg Dinefwr, 2002), t. 171.

${ }^{39}$ Luis Antonio de Villena, 'Teatro de texto y realidad', El Mundo, 27 May, 1994. [fy nghyfieithiad] ${ }^{40}$ Enoch Brater, 'After the Absurd', yn Enoch Brater a Ruby Cohn (goln), Around the Absurd: Essays on Modern and Postmodern Drama (Michigan: University of Michigan Press, 1990), t. 295. ${ }^{41}$ Enrique Centero, “Cambio y comunication en el teatro postfranquista.” El Pais (16 Septembre 1979): viii (“Arte y Pensamiento”) a Carles Batlle i Jordà, 'Contemporary Catalan Theatre: Between the Desert and the Promised Land', Contemporary Theatre Review, 17 (2007), 416-24. Ystyrier hefyd farn Paul Griffiths wrth iddo wylio Diweddgan gan Samuel Beckett er enghraifft; 'Siom arall a methiant drudfawr ac annheilwng i goffáu canmlwyddiant geni Samuel Beckett, a dechrau’r diweddgan go iawn i’r Theatr Genedlaethol. Rhaid newid y gân yn fuan, fuan iawn. Plîs. . ’’ Paul Griffiths, [Ar lein]. Ar gael: http://paulpesda.blogspot.co.uk/search?updated-min=2006-0101T00:00:00Z\&updated-max=2007-01-01T00:00:00Z\&max-results=39, cyrchwyd 19 Medi 2018 ${ }^{42}$ Gwyn Thomas, ‘Tu Hwnt i’r Llen,’ Ysgrifau Beirniadol IX, (1976), 364.

${ }^{43}$ Ibid., 364-5.

${ }^{44}$ Gareth Miles, ‘Bardd y di-sens’, Barn, 545 (Mehefin 2008), 62. 
${ }^{45}$ Cefin Roberts a Mari Emlyn, 'Drama Hir Agored’, yn J. Elwyn Hughes (gol.), Eisteddfod Genedlaethol Blaenau Gwent a Blaenau'r Cymoedd 2010: Cyfansoddiadau a Beirniadaethau, (Llandysul: Gomer, 2010), t. 195.

${ }^{46}$ José Antonio Sánchez, 'The Impossible Theatre’: The Spanish Stage at the Time of the AvantGarde’, cyfieithwyd gan Jill Pythian, Contemporary Theatre Review, 7, (1998), 7-30.

${ }^{47}$ J.L. Styan, addasiad Cymraeg gan Annes Glyn, Y Ddrama Gyfoes: Damcaniaethau ac arferion: Symboliaeth, Swrealaeth a'r Absw'rd (Llandysul: Gwasg Gomer, 2007), tt.152-3.

${ }^{48}$ Martin Esslin, The Theatre of the Absurd, 7fed argraffiad (London: Bloomsbury, 2013), tt. 363-4.

${ }^{49}$ Batlle i Jorda, 'Contemporary Catalan Theatre: Between the Desert and the Promised Land', Contemporary Theatre Review, 416-424.

${ }^{50}$ Robert Morley yn Peter Hall, 'Godotmania’, The Guardian, [Ar lein]. Ar gael: https://www.theguardian.com/stage/2003/jan/04/theatre.beckettat100 , cyrchwyd 1 Hydref 2018 ${ }^{51}$ Bhabha, The Location of Culture, t. 86.

52 Ibid.

53 Jones, Ysbryd y Cwlwm, t. 6.

${ }^{54}$ Aled Jones Williams, Ychydig Is Na'r Angylion (Caernarfon: Gwasg y Bwthyn, 2006), t. 70.

${ }^{55}$ Burghard Baltrusch, ‘The Postmodern Avant-Gardes in Post 1975 Galician Literature: Rompente, Antón Reixa a Suso de Toro’, yn Contemporary Galician Cultural Studies, (2011), 237- 8.

${ }^{56}$ Ibid., 244. 'They try to ensure that Galician cultural and National identity no longer clings to an Aristotelian aesthetic or a simple fraternization between the artistic and public.'

${ }^{57}$ George a London, ‘Avant Garde Drama’, Contemporary Catalan Theatre: An Introduction, t. 91. 'Of all the writers included in this book, Belbel is unique for his exclusive dedication to the theatre, and his independence from the shadow of the Franco era.'

${ }^{58}$ George, Sergi Belbel \& Catalan Theatre, t. 4. 
${ }^{59}$ Belbel yn Feldman, In the Eye of the Storm, t. 171. '[y] si me gustan al mismo tiempo La Fura dels Baus y Molière, no es una contradicción. [fy nghyfieithiad]

${ }^{60}$ George a London, ‘Avant Garde Drama’, Contemporary Catalan Theatre: An Introduction, t. 94.

${ }^{61}$ David George, Sergi Belbel \& Catalan Theatre, t. 197. Gwelir hyn ar waith yn Carícies gan Belbel lle mae modd gweld dylanwad y dramodydd o Awstralia Arthur Schnitzler. Mae i'r ddrama 10 golygfa sy’n ymgysylltu. Mae dau gymeriad yn ymddangos ym mhob golygfa ac mae un o’r ddau gymeriad hynny yn ymddangos yn yr olygfa ddilynol sy’n creu llinyn cyswllt trwy'r ddrama. At hyn, mi fyddai modd ystyried dramâu megis Iesu!, Merched Eira a 'Ta-ra Teresa’ gan Jones Williams yn enghreifftiau o ddramâu sy’n gwthio ffiniau ffurf y ddrama Gymraeg.

${ }^{62}$ Ros, ‘Cyfweliad gydag Aled Jones Williams’, Disgwyl Býs yn Stafell Mam, t. 8.

63 Elan Closs Stepthens, ‘Gramadeg Gwenlyn’, Barn, 465 (Hydref 2001), 55.

${ }^{64}$ Alex Sierz, 'In-Yer-Face-Theatre’, British Drama Today (London: Faber 2001), t. 6. Ystyrier dramâu megis La Sang, Dins la Seva Memòria gan Belbel a Cnawd a Sundance gan Jones Williams fel esiamplau o ddramâu sy’n arddangos yr elfennau amrwd y cyfeirir atynt uchod.

${ }^{65}$ Williams, 'Pêl Goch’, Disgwyl Býs yn Stafell Mam, t. 46.

${ }^{66}$ Cyfieithiad David George yn George, Sergi Belbel \& Catalan Theatre, t. 14.

${ }^{67}$ Williams, 'Wal’, Disgwyl y Býs yn Stafell Mam, t. 101.

${ }^{68}$ Feldman, In the Eye of the Storm, t. 166.

${ }^{69}$ Sergi Belbel cyfieithwyd gan Sharon G. Feldman, 'Fourplay’, yn John London a David George (gol.), Modern Catalan Plays (London: Methuen, 2000), t. 179.

${ }^{70}$ J. Sanchis Sinisterra, 'Sergi Belbel: la pasio de la forma’, Dins La Seva Memòria (Barcelona: Edicions 62, 1988), t. 10.

‘la materia sonora - rítmico y fónica - del lenguaje’ [fy nghyfieithiad]

${ }^{71}$ Williams, 'Wal', Disgwyl y Býs yn Stafell Mam, t. 92. Atgyfnerthir yr amwysedd ynghylch perthynas y cymeriadau â’i gilydd ar ôl sylweddoli bod dwy lythyren flaen enwau'r cymeriadau yn 
ffurfio’r enw Aled. Mae amwysedd ynghylch perthynas cymeriadau â’i gilydd yn rhywbeth a welir yn fynych yn nramâu Belbel hefyd. Ystyrier En Companyia d'amisme, Carícies ac A la Toscana ynghyd â Despres de la Pluja yn enghreifftiau o ddramâu o’r fath.

${ }^{72}$ Aled Jones Williams, Lysh (Caernarfon: Gwasg Gwynedd, 2004), t. 51.

${ }^{73}$ Idem., Oerfel Gaeaf Duw (Caernarfon: Gwasg Pantycelyn, 2002), t. 43.

${ }^{74}$ Jaques Lacan yn Bhabha, The Location of Culture, t. 85.

${ }^{75}$ Belbel, ‘Fourplay’, Modern Catalan Plays, t. 181,

${ }^{76}$ Aled Jones Williams, Anweledig, Cwmni’r Fran Wen, Copi anghyhoeddedig o’r sgript.

${ }^{77}$ Gruffudd Eifion Owen, 'Saunders, Kate ac Aled', [Ar lein]. Ar gael:

http://cylchgrawnbarn.com/index.php?option=com_content\&view=article\&id=413:saunders-kate-acaled\&catid=34:erthyglau\&Itemid=92, 12 Medi 2018

'Mae Aled Jones Williams yn feistr ar greu troeon ymadrodd a delweddau trawiadol, ac mae Anweledig yn frith ohonynt.'

${ }^{78}$ Sergi Belbel, Dins la Seva Memòria, t. 32. ‘ «Ell», per primera vegada ell era «ell». «Ell» una paraula que començaries a utilizar a partir de llavors.' Mae hyn yn dwyn i gof darn o 'Wal' wrth i Alji son am y profiad prin yna o deimlo ei fod yn adnabod ei hun. Williams, 'Wal', Disgwyl y Býs yn Stafell Mam, t. 102.

${ }^{79}$ Maria M. Delgado a David George, ‘Sergi Belbel’, yn Mary Parker (gol.), Modern Spanish Dramatists: A Bio-Bibliographical Sourcebook (London: Greenwood, 2002), t. 78.

${ }^{80}$ Luned Emyr, ‘Mynd i gyfeiriad arall’, cyfweliad ag Aled Jones Williams yn Taliesin, 128 (2006), 119.

${ }^{81}$ Ystyrier monolog Lucky, er enghraifft, yn Samuel Beckett, 'Waiting for Godot', Samuel Beckett the Complete Dramatic Works (London: Faber and Faber, 2006), tt. 42-3.

${ }^{82}$ Williams, 'Pêl Goch', Disgwyl y Býs yn Stafell Mam, t. 66.

${ }^{83}$ Belbel, A la Toscana, (Barcelona: Proa, 2007), t. 41. 
Marc: M'estimes?

Joana: Sí.

Marc: És veritat?

Joana: Què importa, are, si es veritat? T'estàs morint. L'important es que t'ho digui. I que t’ho creguis.

Marc: Sí. (Pausa.) M’ho creuré. És l’unic consol, oi? (Pausa.) Digue-m’ho.

Joana: T’estime. [fy nghyfieithiad]

${ }^{84}$ George, Sergi Belbel, t. 30.

85 John Gwilym Jones, 'Drama hir: beirniadaeth John Gwilym Jones’, yn T.M. Bassett, Eisteddfod Genedlaethol Frenhinol Cymru Maldwyn a’i Chyffiniau 1981: Cyfansoddiadau a Beirniadaethau 1981 (Llandysul: Gomer, 1981), t. 150. ‘Gellir, fel Morgan Llwyd, ymhyfrydu yng nghoethder geiriau, neu, fel T. H. Parry-Williams, ymfoethuso yn agosatrwydd tafodiaith, neu, fel Saunders Lewis, pan fo’r galw, eu trin fel cyllyll i frifo.'

${ }^{86}$ Sergi Belbel, Morir (un momento antes de morir) (Madrid: Ant Machado Libros, 2014), t. 28. Hija: Que los chicles no quitan el hambre porque ayer estuve comiendo chicles todo el dia y tenía un hambre que me moria.

Madre: ¿Y hoy que es lo que te pasa?

Hija: Nada. Que quiero hacer regimen. Una amiga me ha dicho que la obesidad es hereditaria. (La madre está a punto de abofetar a la Hija.) [fy nghyfieithiad]

${ }^{87}$ Feldman, 'Post-Franco Theatre', The Cambridge History of Spanish Literature, t. 726.

${ }^{88}$ Tudur Hallam, Saunders y Dramodydd (Caernarfon: Gwasg Pantycelyn, 2013), t. 75.

${ }^{89}$ Aled Jones Williams, Lysh (Caernarfon: Gwasg Gwynedd, 2004), t. 34.

${ }^{90}$ Belbel, cyf. London, 'Caresses’, Spanish Plays: New Spanish and Catalan Plays, goln. Elyse Dogson a Mary Peate (London: Nick Hern Books, 1999), t. 5.

${ }^{91}$ Williams, ‘Ta-ra Teresa’, Disgwyl býs yn Stafell Mam, tt. 154-5. 
92 Aled Jones Williams, 'Palimpsest', Y Cylchoedd Perffaith, t. 79.

${ }^{93}$ Belbel, A la Toscana, tt. 12-3.

${ }^{94}$ Gwyn Griffiths, 'Deuawd Aled Jones Williams’, [Ar lein]. Ar gael:

http://www.bbc.co.uk/cymru/eisteddfod/2010/safle/ormaes/bara-caws.shtml, cyrchwyd 13 Medi

2018.

${ }^{95}$ Williams, 'Merched Eira', Merched Eira a Chwilys, t. 34 a David George, Sergi Belbel, tt. 39-40.

${ }^{96}$ Aled Jones Williams, yn Williams, 'Holi Aled Jones Williams’, Ysgrifau Beirniadol XXIX, 20.

${ }^{97}$ Feldman, 'Post-Franco Theatre', The Cambridge History of Spanish Literature, t. 735.

${ }^{98}$ Ros, 'Rhwng rheg a thethreg’ Disgwyl Býs yn Stafell Mam, t. 20.

${ }^{99}$ Kirsti Bohata, Postcolonailsm Revisited (Cardiff: University of Wales Press, 2004), t. 105. 100 Ibid.

${ }^{101}$ PepBlay, 'Entrevista: Sergi Belbel', Avui Diumenge, 24 February 1991, 6 a Belbel, 'Deep Down’, cyf. John London, Modern International Drama, 26, (1993), 9. Gwelir hyn ar waith eto yn Despres de la pluja wrth iddo gyfosod iaith bob dydd ac iaith lenyddol. Mae George yn trafod hyn gan ddadlau fod hyn yn enghraifft arall o’r modd y mae Belbel yn defnyddio ei sensitifrwydd at iaith i anesmwytho’r gynulleidfa yn George, Sergi Belbel, t. 16.

102 Peter Conrad, 'But You’ll Have to Wait for Godot', Observer, review section, 24 May 2009, 20 yn George, Sergi Belbel, t. 15. Astudiodd Belbel ieitheg Ffrangeg cyn troi at fyd y ddrama.

${ }^{103}$ Cymh. Robert Rhys, ‘Dysgu Darllen’ yn John Rowlands (gol.), Sglefrio ar Eiriau, (Llandysul: Gomer, 1992), t. 165: ‘Rhoddwyd pwyslais gormodol ar ystyriaethau cofiannol neu fywgraffyddol ym maes astudiaethau llenyddol, ond weithiau fe fydd gwybodaeth o'r fath yn angenrheidiol.' A R. M. Jones, Beirniadaeth Gyfansawdd (Abertawe: Cyhoeddiadau Barddas, 2003), t. 234: 'cofier y gall arferion 'all-llenyddol' hefyd fod yn ddigon perthnasol.'

104 ‘Aled Jones Williams yn Gadael yr Eglwys’, [Ar lein]. Ar gael: http://www.bbc.co.uk/cymru/bywyd/safle/crefydd-straeon/tudalen/aledjw-gadael.shtml, cyrchwyd 6 
Hydref 2018. At hyn, gellid ychwanegu sylwadau Williams at yr ymateb chwyrn a fu gan rai beirdd mwy ceidwadol at y bryddest a enillodd iddo’r goron yn Eisteddfod Genedlaethol Tyddewi 2002. Yn sgil yr ymateb hwnnw esboniodd Williams nad oedd ef yn teimlo’n rhan o'r cylch hwnnw a’i fod yn teimlo ar gyrion pethau. 'Prifardd nad yw’n un o’r “giang”, [Ar lein]. Ar gael: http://www.bbc.co.uk/cymru/adloniant/llyfrau/straeon/021010aled.shtml , cyrchwyd 30 Hydref 2018 ${ }^{105}$ Williams, 'Holi Aled Jones Williams’, 10-2.

${ }^{106}$ Williams, Y Cylchoedd Perffaith, t. 38.

${ }^{107}$ Williams, ‘Holi Aled Jones Williams’, Ysgrifau Beirniadol XXX1, 10-1.

${ }^{108}$ Ibid., t. 12.

${ }^{109}$ Williams, ‘Alcoholic', Y Cylchoedd Perffaith, t. 34.

${ }^{110}$ Williams, ‘Pryd Fuo Kathleen Ferrier Farw?’, Disgwyl býs yn Stafell Mam, t. 131.

${ }^{111}$ Yn wir, y mae Albert Bastardas i Boada, Emili Boix-Fuster, Rosa M. Torrens Guerrini (goln.), El Català, Llengua Mitjana D’Europa (Barcelona: Octaedro, 2018) yn dadlau nad iaith leiafrifol mo’r Gatalaneg bellach gan fod 73\% o’r boblogaeth yn siarad Catalaneg a 95\% yn ei deall ymhlith rhesymau eraill. [Ar lein]. Ar gael: https://www.nationalia.info/new/10063/census-reveals-73-speakcatalan-in-catalonia-95-understand-it-56-can-write-it, cyrchwd 20 Awst 2018

112 Owen, 'Y dieithredig’, Barn, 94.

${ }^{113}$ Ros, 'Rhwng Rheg a Rhethreg', t. 33, ‘Roedd cyffro noson gyntaf Ta-ra Teresa yn annodweddiadol i’r theatr Gymraeg, gyda’r edrych ymlaen eiddgar yn amlwg yn y gynulleidfa. Roedd y rhai a ddisgwyliai neges gref, a gwrth-Saesneg, wedi'u siomi, ond roedd yna fwyafrif yn teimlo eu bod yn bresennol mewn digwyddiad theatrig o bwys: drama fawr ar thema fawr.' ${ }^{114}$ Williams, 'Pêl Goch', Disgwl Bỳs yn Stafell Mam, t. 59.

${ }^{115}$ Idem., ‘Ta-ra Teresa’, Disgwyl y Býs yn Stafell Mam, t. 160.

${ }^{116}$ Huw Roberts, 'Dramodydd yr isfyd', Barn, 410 (Mawrth 1997), 28. 
${ }^{117}$ Williams, Ta-ra Teresa', Disgwyl y Býs yn Stafell Mam, tt. 150-1. Ceir sawl cyfeiriad at Dryweryn e.e. 'ROBART HEFIN: Be oedd Tryweryn, Nhad? Medda fi wrtho fo ar y býs. Nid be ond lle. Lle ddaeth yn egwyddor, medda fynta. Fedrwn i ddim dallt yn wyth oed pam oedd raid boddi cwm yng Nghymru i roid dŵr i bobol mewn tre mor bell i ffwr' o'r enw Lerpwl. O'dd 'na nunlla'n nes, Nhad? medda fi. Pam, Nhad? medda fi wrtho fo drachefn. Am’n bod ni wedi ca’l ein g’neud i gael ein sathru, medda fo’n sbïo ar Mam.’

${ }^{118}$ Williams, ‘Ta-ra Teresa’, Disgwyl y Býs yn Stafell Mam, tt. 156-7. Gellir gweld sawl tebygrwydd rhwng Adrian a chymeriad Nerys yn nofel Aled Jones Williams, Eneidiau (Llanrwst: Gwasg Carreg Gwalch, 2013).

${ }^{119}$ Ibid., tt. 177-8.

${ }^{120}$ Ibid.

${ }^{121}$ Williams, Tuchan o Flaen Duw, t. 78.

${ }^{122}$ Belbel, ‘Deep Down’, cyf, London, Modern International Drama, t. 11.

${ }^{123}$ http://anffyddiaeth.blogspot.co.uk/2015/07/cymru-dal-i-gredu.html, cyrchwyd 4 Gorffennaf 2018 https://twitter.com/hashtag/DaliGredu?src=hash, cyrchwyd 4 Gorffennaf 2018.

Er bod 56\% o boblogaeth Cymru wedi nodi eu bod yn Gristnogion, 14.3\% yn llai nag y nodwyd yng nghyfrifiad 2001. Dengys cyfrifiad 2011 hefyd fod yng Nghymru y nifer uchaf o bobl sy’n dweud eu bod yn anffyddwyr. [Ar lein]. Ar gael:

https://www.ons.gov.uk/peoplepopulationandcommunity/culturalidentity/religion/articles/religionine nglandandwales2011/2012-12-11 ,cyrchwyd 20 Awst 2018.

${ }^{124}$ Aled Jones Williams, Oerfel Gaeaf Duw (Caernarfon: Gwasg Pantycelyn, 2002), t. 22. Ceir trafodaeth bellach ar hyn a pherthynas Aled Jones Williams â'r gair 'Duw' yn Aled Jones Williams a Cynog Dafis, Duw yw’r Broblem (Llanrwst: Gwasg Carreg Gwalch, 2016), t. 14: ‘A dyna erbyn heddiw sy’n anodd: anadlu ‘Duw’ mewn aer nad yw bellach yn medru cynnal ‘Duw’ - mae’r awyrgylch i’r gair bellach ffynnu ynddo yn ein gwlad yn denau ac yn teneuo.' 
${ }^{125}$ Williams, 'Pêl Goch’, Disgwyl y Býs yn Stafell Mam, t. 45.

${ }^{126}$ Ros, 'Rhwng rheg a rhethreg', Disgwyl y Býs yn Stafell Mam, tt. 9-10.

${ }^{127}$ Belbel, Morir (un instantes antes de morir), t. 58. ASESINO: Cállese. A ver. . . me ha dicho que no le mate. . . no le mate por. . . Dios. Por. . . Dios. Por. . . Dios, ¿verdad? Muy bien, este as el reto: usted llama a Dios y le pide que venga y que le salve. ¿De acuerdo? Porque usted cree ên él, ¿̇no es cierto?

VICTIMA: Sí. Usted también.

ASESINO: Yo? No sé. Mire, si se me aparece ên cinco minutos y me impide que le mate, creeré en el. Por supuesto que si. Hala, vamos a empezar. [fy nghyfieithiad] ${ }^{128}$ Ros, ‘Cyfweliad gydag Aled Jones Williams’, Disgwyl y Býs yn Stafell Mam, t. 15. Aled Jones Williams: '[doeddwn i ddim yn hapus o gwbl efo Robert Hefin. Doeddwn i ddim yn siŵr iawn sut i’w achub o a dydw i'n dal ddim. Hynny ydy, roedd o'n mynd yn fwy o ystrydeb Cymdeithas yr Iaith, ac er pob dim oeddwn i’n drio’i wneud efo fo roedd o’n dal i fod felly. A hwyrach mai ysgrifennu Robat Hefin oeddwn i ac nid mynd i mewn i gymeriad Robert Hefin. Oherwydd dydy’r profiad o'r math yna o genedlaetholwr ddim gen i, yr unig beth gen i oedd y gwawdlun. Felly dwi'n meddwl mod i 'di ysgrifennu gwawdlun yn fanna.'

${ }^{129}$ Xavier Puchades, 'Renovacion Teatral en España entre 1984-1998 desde la escritura dramática; puesta en escena y receptión crítica’, Traethawd doethurol angyhoeddedig, Prifysgol Valencia 2005, t. 72 .

${ }^{130}$ Feldman, In the Eye of the Storm, t. 25. 'In democratic times, the presence of the Catalan language as a vehicle of theatrical expression has continued to hold symbolic value as a sign of identity and a vindication of Catalan culture. For many contemporary Catalan dramatists, innately conscious of the opressive realities of the past, the linguistic distinction appears to take presedence over all other thematic or assthetic indicators of identity. To write and/or to stage a play in Catalan is, 
in effect, to inscribe and reclaim a specific cultural space.' Saunders Lewis, Egwyddorion Cenedlaetholdeb (Caerffili: SWP cyf.), t. 14.

${ }^{131}$ Sergi Belbel, 'On Writing, Directing and the Teatre Nacional de Catalunya’, Contemporary Theatre Review, Vol. 17, (2007), 415.

${ }^{132}$ Williams, 'Holi Aled Jones Williams’, 10-1. O ran Sergi Belbel mae David George a John London yn nodi hyn: 'Sergi Belbel has had no great problems being accepted either as an avantgarde dramatist or a Catalan writer, in spite of his Spanish-speaking family background.' George a London, ‘Avant-garde Drama’, Contemporary Catalan Theatre: An Introduction, t. 91.

${ }^{133}$ Sergi Belbel, cyfieithiad Marion Peter Holt, Blood (New Brunswick: Estreno), t. 9.

${ }^{134}$ Belbel, Guillermo Heras, Jose Sanchis Sinisterra, et al, "Perspectivas dramaturgicas: Hacia el siglo XXI”, Transcript of PANEL DISCUSSION. Sitges Teatre International 1998. Escena 60-61 (May-June 1999)

${ }^{135}$ Feldman, In the Eye of the Storm, tt. 40-1.

${ }^{136}$ Carles Batlle i Jordá, “La realitad i el joc”, Pausa, 20 (January 2005), 71.

${ }^{137}$ George, Sergi Belbel, tt. 119- 58. Rhoddodd Sharon Feldman bapur dan y teitl, 'Toward a New Cosmopolitanism in Contemporary Catalan Drama’ fel rhan o'r symposiwm (Dis)articulating Identities: Multilingualism in the Catalan Countries, a gynhaliwyd ym Mhrifysgol Caergrawnt, 19 Ionawr 2018

${ }^{138}$ Helena Buffery, 'Contemporary Catalan Theatre Identity: The Haunted Mirrors of Catalan Directors', Theatre and Performance in Small Nations, t. 78.

139 Ibid.

${ }^{140}$ Gerallt Lloyd Owen, 'Fy Ngwlad’ yn Blodeugerdd o Farddoniaeth Gymraeg yr Ugeinfed Ganrif, goln. Gwynn ap Gwilym ac Alan Llwyd (Llandysul: Gomer, 1987), t. 507.

${ }^{141}$ Aled Jones Williams, Pridd (Llanrwst: Gwasg Carreg Gwalch, 2013), t. 13. 
142 J. Sanchis Sinisterra, 'Sergi Belbel: la pasio de la forma’, Dins La Seva Memòria, t. 9. ‘[u]n àmbit oscur que no pretén representar res, que no aspira a figurar altra cosa que el que es: l'escenari.’ [fy nghyfieithiad]

143 Aled Jones Williams, Merched Eira a Chwilys, t. 20.

${ }^{144}$ Enric Gallén, ‘A la Toscana o la felicitat fregmentada’, A la Toscana, t. 12.

${ }^{145}$ Belbel, Dins la Seva Memòria, t. 22.

3: Ara

2: ja ha arribat el moment.

3: Es ara el moment.

1: Quan aquest temps

2: ja t'ha paralitzat

3: i consumit.

2: Molts dies!

3: Tants dies

1: Pots comptar-los.

2: Molts

3: Tants dies.

${ }^{146}$ Ibid., t. 78.

${ }^{147}$ Manon Wyn Williams, ‘Tri Dramodydd Cyfoes: Meic Povey, Siôn Eirian ac Aled Jones Williams’, Traethawd PhD anghyhoeddedig, Prifysgol Bangor, 2015, tt. 275-82.

${ }^{148}$ Ibid a Ros, 'Rhwng rheg a rhethreg', Disgwyl y Býs yn Stafell Mam, t. 17. Cerddi sy’n corddi ydy cynnyrch Aled Jones Williams. Y cyfuniad o regfeydd a rhyw, agnosticiaeth ac anffyddiaeth, sydd yn gyfrifol am gyflwr myfyrgar a holgar ei gynulleidfaoedd a’i ddarllenwyr. Mae'r codi cwestiynau weithiau’n codi gwrychyn am nad oes ateb yn cael ei gynnig. ${ }^{149}$ Aled Jones Williams, Iesu! (Llandysul: Gwasg Gomer, 2008), t. 40 . 
${ }^{150}$ Williams, ‘Tri Dramodydd Cyfoes: Meic Povey, Siôn Eirian ac Aled Jones Williams’, t. 265.

${ }^{151}$ Williams yn Ros, ‘Cyfweliad gydag Aled Jones Williams’, Disgwyl y Býs yn Stafell Mam t. 11.

152 Williams, Y Cylchoedd Perffaith, t. 67.

${ }^{153}$ Edwards, [Ar lein]. Ar gael: http://www.bbc.co.uk/cymru/adloniant/theatr/adolygiadau/iesu.shtml , cyrchwyd 24 Medi 2016

154 Jones, Ysbryd y Cwlwm, t. 11.

${ }^{155}$ Blandford, 'Drama Fictions in a Postcolonial Wales’, yn Jane Aaron a Chris Williams (goln.), Postcolonial Wales (Cardiff: Cardiff Unibersity Press, 2005), t. 191.

${ }^{156}$ Ed Thomas yn Ibid.

${ }^{157}$ Cyfeiria Blandford at sylwadau Ian Rowlands am y Theatr Genedlaethol yn Blandford, 'Theatre and Performance in a Devolved Wales', Theatre \& Performance in Small Nations, t. 65.

Paul Griffiths, Theatr Genedlaethol Cymru, [Ar lein]. Ar gael:

http://paulpesda.blogspot.com/2009/05/theatr-genedlaethol-cymru.html, cyrchwyd 18 Hydref 2018. 158 Josep-Anton Fernàndez, El Malestar en la cultura Catalana: La Cultura de Normalizació 19761999 (Barcelona: Editorial Empúries, 2008)

159 Ibid.

${ }^{160}$ Ian Brown, 'Process and Interactive Events: Theatre and Scottish Devolution', Theatre \& Performance in Small Nations, t. 40.

${ }^{161}$ Alun Lewis yn Steve Blandford, 'Theatre and Performance in a Devolved Wales', Theatre and Performance in Small Nations, t. 65. 